\title{
Design of Complex Oxide Interfaces by Oxide Molecular Beam Epitaxy
}

\author{
Y. E. Suyolcu ${ }^{1}$ (D) G. Christiani ${ }^{1}$ - P. A. van Aken ${ }^{1} \cdot$ G. Logvenov ${ }^{1}$
}

Received: 2 July 2019 / Accepted: 28 August 2019 / Published online: 21 October 2019

(C) The Author(s) 2019

\begin{abstract}
Complex oxides provide a versatile playground for many phenomena and possible applications, for instance, high-temperature superconductivity, magnetism, ferroelectricity, metal-to-insulator transition, colossal magnetoresistance, and piezoelectricity. The origin of these phenomena is the competition between different degrees of freedom such as charge, orbital, and spin, which are interrelated with the crystal structure, the oxygen stoichiometry, and the doping dependence. Recent developments not only in the epitaxial growth technologies, such as reactive molecular beam epitaxy, but also in the characterization techniques, as aberration-corrected scanning transmission electron microscopy with spectroscopic tools, allow synthesizing and identifying epitaxial systems at the atomic scale. Combination of different oxide layers opens access to interface physics and leads to engineering interface properties, where the degrees of freedom can be artificially modified. In this review, we present different homo- and hetero-epitaxial interfaces with extraordinary structural quality and different functionalities, including hightemperature superconductivity, thermoelectricity, and magnetism.
\end{abstract}

Keywords Oxide molecular beam epitaxy $\cdot$ Complex oxides $\cdot$ High-temperature superconductivity $\cdot$ Hetero-structure interfaces . Scanning transmission electron microscopy

\section{Introduction}

Complex oxide hetero-structures create a multidirectional platform for the emergence of significant properties stemming from the strong interplay between the lattice, spin, orbital, and charge degrees of freedom $[1,2]$. The easily modifiable crystal structure and tailorable oxygen sublattice of the generally studied $\mathrm{ABO}_{3}$ perovskite structure, which is composed of corner-sharing $\mathrm{BO}_{6}$ octahedra and bodycentered A-site cations surrounded oxygen anions, bring the advantage of fine-tuning properties through picometerscale modifications in the lattice [3]. Especially after the discovery of high-temperature superconductivity [4], the epitaxial growth of cuprate-based systems such as $\mathrm{La}_{2}$ ${ }_{-x} \mathrm{Sr}_{x} \mathrm{CuO}_{4}$ (LSCO) [5, 6], $\mathrm{YBa}_{2} \mathrm{Cu}_{3} \mathrm{O}_{7-\delta}$ (YBCO) [7-9],

G. Logvenov

g.logvenov@fkf.mpg.de

Y. E. Suyolcu

y.e.suyolcu@fkf.mpg.de

1 Max Planck Institute for Solid State Research, Heisenbergstrasse 1, 70569 Stuttgart, Germany
$\mathrm{DyBa}_{2} \mathrm{Cu}_{3} \mathrm{O}_{7-\delta}$ (DBCO) [10], $\mathrm{Bi}_{2} \mathrm{Sr}_{2} \mathrm{Ca}_{n-1} \mathrm{Cu}_{n} \mathrm{O} 2_{n+4}$ (BSSCO) [11], received a strong interest, together with the other functional oxide compounds with different functionalities [12-14]. In addition to the synthesis of single-phase films, the epitaxial growth of such complex oxides in the form of multilayered systems further yields the emergence of novel interfacial phenomena [15-17], which are not present in the constituent layers alone. These systems provide not only a wide range of applications with prominent functionalities such as ferroelectricity [18, 19], emergence of 2D electron gas [20], thermoelectricity [21], magnetism [22-24], and superconductivity [25], but also understanding the fundamental nature of the underlying complex phenomena. The quality of the multicomponent oxide thin films can be defined as a function of the stoichiometry, the structural quality and the extent of present defects, whereas the interface sharpness has been realized as the most critical factor for novel applications. An intriguing example is the hightemperature interface superconductivity (HT-IS) emerging at the interface between non-superconducting undoped and overdoped copper oxide layers [26], for which the absence of a specific accepted theory of high-temperature superconductors (HTS) fosters the strong interest. 
In general, the precision of the epitaxial growth techniques determines the limits of the synthesis and accordingly the control and the understanding of the materials' properties. Recent technical developments in epitaxial growth technologies pave the way for synthesizing high-quality oxide heterostructures with atomic layer precision. Different physical deposition techniques, such as pulsed laser deposition (PLD) [27-31], reactive sputtering, and reactive molecular beam epitaxy (MBE) with in situ reflection high-energy electron diffraction (RHEED) [32-37], have been comprehensively utilized. The reactive ozone-assisted MBE can be considered as the most powerful technique, since it allows high-quality fabrication with great variability, low-energy of impinging atoms, and layer-precise control of the growth [38], which brings advantages on structural quality and interface sharpness [39]. The combination of such unique deposition methods with at least one foremost characterization technique is an inevitable step for further progress in the field of oxide hetero-structure engineering, especially for interface-focused research. In particular, cuprate-based hetero-interfaces are impressive systems with the possibility to emerge the superconducting state, which is substantially sensitive to material characteristics, e.g., film stoichiometry and the presence of defects (impurities, oxygen vacancies). Complimentary characterization methods such as in situ RHEED [40-43], atomic force microscopy (AFM), high-resolution X-ray diffraction [44, 45], together with different spectroscopic methods [46-53], identify the crystal quality, the surface roughness, the stoichiometry, and the electronic structure. Additionally, measurements of superconducting transitions via the two-coil mutual inductance [26] or the electrical resistance further reflect the quality of the MBE synthesis. Consequentially, the atomic-layer precise fabrication of these systems requires atomic-resolution identification. In this context, one prominent tool for interface characterization is aberration-corrected scanning transmission electron microscopy (STEM) [54-56] with atomic-resolution imaging and spectroscopy capabilities.

In this work, we present a brief overview of different complex oxide hetero-structures fabricated by ozone-assisted MBE (Fig. 1a) with precise atomic layer control, i.e., $\mathrm{La}_{2} \mathrm{CuO}_{4}, \mathrm{La}_{2-x} \mathrm{~A}_{x} \mathrm{CuO}_{4}(\mathrm{~A}=\mathrm{Ca}, \mathrm{Sr}, \mathrm{Ba}), \mathrm{La}_{2-x} \mathrm{Sr}_{x} \mathrm{NiO}_{4}$, $\mathrm{LaNiO}_{3}$, and $\mathrm{SrMnO}_{3}-\mathrm{LaMnO}_{3}$, in addition to the comprehensive recent achievements in HT-IS in LCO-based systems. The aberration-corrected STEM (Fig. 1b) with the positionsensitive atomic-resolution characterization capability was used to determine the interfacial quality. This review is structured as follows: In Chapter 2, we present the growth and characterization of high-quality single-phase $\mathrm{La}_{2} \mathrm{CuO}_{4}$ (LCO) films together with underlining the importance of the oxygen content as well as a way to handle oxygen exchange dynamics through high-temperature consideration of the electrical properties. In Chapter 3, we overview the homoepitaxial HT-IS including previous results from the
Brookhaven group as well as our recent studies focusing on the influence of the cationic radii on the interface sharpness of metal-insulator bilayers and associated physical properties, as well as the asymmetric distribution of the cations across the $\mathrm{Sr}$ delta-doped layer in a LCO matrix. The hetero-epitaxial cuprate-nickelate and cuprate-manganite interfaces are described in Chapter 4, with considerations of HT-IS, layer thickness dependence of electrical properties, and the design of superconducting-magnetic individual interfaces, respectively. Finally, the summary and outlook are presented in Chapter 5.

\section{Single-Phase Films}

The initial challenge in epitaxial complex oxide heterostructures is to satisfy the sample quality, since various parameters jointly determine the crystal structure, the interface sharpness/roughness, and the correlated properties of these systems. Hence, the first step is to achieve high-quality single-phase epitaxial films. Herewith, we briefly touch on the critical parameters for the oxide thin film growth. The fundamental distinction of the epitaxial films from bulk single crystals is the presence and the explicit involvement of the substrate, which points the structural properties of the epitaxial films. The choice of the substrate can yield different properties by modifying the crystal structure of the thin epitaxial films via inducing small lattice distortions [57, 58]. From one side, the lattice mismatch between the epitaxial film and the substrate should be relatively small and favorable for forming a crystal with the minimum number of defects, for instance, oxygen vacancies [59,60] and misfit dislocations [61, 62]. On the other side, the substrate-induced strain should be suitable for creating novel functionalities and possible improvements. Furthermore, the oxidation conditions, namely the substrate temperature and the oxidant pressure, which mutually relate with the stoichiometry and/or the composition control, play a crucial role in the correct phase formation as well [63]. The stoichiometry control during the growth is challenging due to the multicomponent composition and requires the management of each atomic flux with high precision to avoid any secondary phase precipitate formation. The initial layer formation at the interface with the substrate and the sequence of the deposited atomic layers should also be taken into consideration in the layer-by-layer MBE growth and for real-time observation of the growth, RHEED has been mainly utilized, which provides primary information about the crystal quality of surface layers and the presence or absence of secondary phase precipitates.

Let us consider the MBE synthesis of hole-doped $\mathrm{La}_{2}$ ${ }_{-x} \mathrm{~A}_{x} \mathrm{CuO}_{4}$ (LACO) epitaxial thin films, which are classical HTS systems with a single $\mathrm{CuO}_{2}$ plane separated by the charge reservoir La-O blocks in the elementary unit cell, 
Fig. 1 Atomic-layer precise oxide MBE and aberrationcorrected STEM. a Dual-chamber oxide MBE system (DCA instruments) of the Thin Film Technology Facility at the Max Planck Institute for Solid State Research [38]. b Aberrationcorrected scanning transmission electron microscope (JEOL JEMARM200CF) of the Stuttgart Center for Electron Microscopy at the Max Planck Institute for Solid State Research [56]
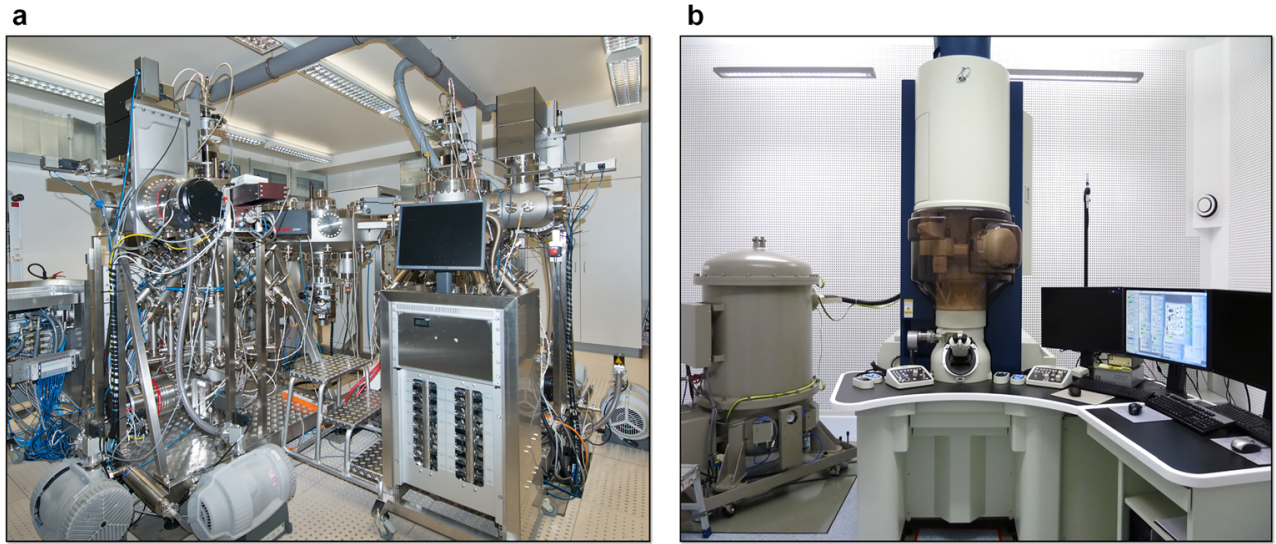

where $x$ indicates the concentration of aliovalent $\mathrm{A}^{2+}$ dopant ion. Advantageously, the wide doping range of the system leads to exploring the complete phase diagram from the undoped insulating parent $\mathrm{La}_{2} \mathrm{CuO}_{4}$ compound with antiferromagnetic order (AFM) to the overdoped nonsuperconducting metallic compound [64, 65]. The carrier concentration in this system is regulated by the substitution of $\mathrm{La}^{3+}$ ion by the dopant ions (e.g., $\mathrm{Ca}^{2+}, \mathrm{Sr}^{2+}, \mathrm{Ba}^{2+}$ ) and/or by the intercalation of oxygen interstitials. The superconducting dome for $\mathrm{Sr}$ - and Ba-doped LCO is obtained within a certain concentration range of hole doping, $p$, i.e., $0.06 \leq p \leq 0.26$ and the highest $T_{c}(\approx 35 \mathrm{~K})$ is realized in the optimum-doped compound with the hole concentration of $p \approx$ 0.16 [66-68]. Note that the concentration of the dopant, $x$, is not exactly equal to the hole carrier concentration, $p$. The ratio between $p$ and oxygen concentration depends on the corresponding formation energies, and the formation of oxygen vacancies can compensate the increase of the dopant concentration, especially in the overdoped regime [69]. While the doping concentration of $\mathrm{Sr}$ and $\mathrm{Ba}$ ions can be varied within a wide range $(0 \leq x \leq 1.4)$ [70], the solubility limit, $x$, of $\mathrm{Ca}$ as dopant in $\mathrm{La}_{2-x} \mathrm{Ca}_{x} \mathrm{CuO}_{4}$ (bulk polycrystalline) is restricted to $x \approx 0.1$ and the maximum superconducting transition temperature achieved is $T_{c} \leq 20 \mathrm{~K}[71,72]$. In the case of thin films, for optimal doping $(x \approx 0.16)$ of Sr- and Ba-doped thin films, the highest $T_{c} \sim 50 \mathrm{~K}$ has been reported, when the films are deposited on $\mathrm{LaSrAlO}_{4}$ (LSAO) substrates [6, 73-75]. A massive number of reports have been devoted to $\mathrm{Sr}$ - and $\mathrm{Ba}$-doped LCO epitaxial films; however, a comprehensive study of Cadoping in epitaxial LCCO films is still missing, while the only successful initiation of $\mathrm{Ca}$ doping in overdoped-undoped (LCCO-LCO) bilayers has been reported [76].

Concerning the substrate-induced strain and $T_{c}$ relationship, due to the lattice constant mismatch between the LSCO film and the LSAO $(001)$ substrate $\left(a_{\mathrm{LSAO}}=3.755 \AA\right)$, which is $0.5 \%$ smaller than the lattice constant of the Sr optimumdoped bulk LSCO $(001)\left[\left(a_{\mathrm{LSCO}}=3.777 \AA\right)\right]$, the film is under compressive strain that results in the highest $T_{c} \sim 45 \mathrm{~K}$. On the other hand, for instance, $\mathrm{SrTiO}_{3}\left(\mathrm{STO}, a_{\mathrm{STO}}=3.905 \AA\right)$ substrate, which has a 3\% larger in-plane lattice constant, induces tensile strain to the optimum-doped LSCO epitaxial film and $T_{c}$ is significantly reduced down to $\sim 20 \mathrm{~K}[70,77]$. Another criterion, heavily affecting the final properties of complex oxides, is the contribution of oxygen and related species, e.g., oxygen vacancies and oxygen interstitials [78-83]. For example, oxygen interstitials can play the role of dopants and contribute to the conductivity and superconductivity in LCO-based compounds, while the formation of oxygen vacancies suppresses superconductivity. Recently, the experimental evidence of the increase of oxygen vacancies in the apical positions (of MBE-grown LSCO films), after lowtemperature vacuum annealing and their variation as a function of Sr doping in highly oxidation environment, has been reported by using confocal Raman spectroscopy [84].

Considering the present understanding and taking the advantage of the atomic-layer precise MBE growth, we have synthesized LCO-based compounds with different dopants $\left(\mathrm{Ca}^{2+}, \mathrm{Sr}^{2+}, \mathrm{Ba}^{2+}\right)$ and varying doping concentration on LSAO substrates, which favors the highest $T_{c}$. Note that $\mathrm{Ca}^{2+}, \mathrm{Sr}^{2+}$, and $\mathrm{Ba}^{2+}$ ions have the same valence state but different ionic radii, i.e., $118 \mathrm{pm}, 131 \mathrm{pm}$, and $147 \mathrm{pm}$, corresponding to a ninefold coordination [85], respectively. The resultant mismatch with the host $\mathrm{La}^{3+}$ cation having an ionic radius of $121 \mathrm{pm}$ is $-2.47 \%,+8.3 \%$, and $+21.5 \%$, which consequently provides different chemical pressures in the crystal structure. In the case of $\mathrm{Ba}$ doping, single-phase LBCO epitaxial films grown on LSAO (001) substrates are characterized by rough surfaces with the presence of secondary phase precipitates containing excess $\mathrm{Ba}$, as confirmed by Auger electron spectroscopy (AES), and also the critical thickness of the nucleation exhibits a linear dependency on $\mathrm{Ba}$ concentration [76]. The best results obtained for Sr-doped LCO epitaxial films grown by our MBE system are comparable with results from other groups $[5,6,70,86]$. Furthermore, we have successfully synthesized precipitate-free $\mathrm{Ca}$-doped LCO epitaxial films on LSAO (001) substrates with broad doping range up to $x=0.6$ and $T_{c} \approx 35 \mathrm{~K}$ at the optimum doping concentration with $x=0.16$. The related report will be 
published elsewhere. The stabilization of Ca-doped LCO films and the segregation of $\mathrm{Ba}$ on the topmost surface result from the interplay between compressive strain induced by the LSAO substrate and the chemical pressure due to the size of dopant ions.

Figure 2a shows an example RHEED image after a completed deposition of $\mathrm{La}_{2} \mathrm{CuO}_{4}$ unit cell showing a characteristic surface reconstruction pattern. Real-time control of RHEED pattern is used for fine control of the growth, in particular the stoichiometry and surface roughness. The transport measurements of a 30 monolayers (MLs) thick optimumdoped LSCO film, i.e., the electrical resistance and the imaginary part of the mutual inductance, as a function of temperature are displayed in Fig. 2b. The superconducting transition (blue) is very sharp, $T_{c}(R=0) \approx 43 \mathrm{~K}$ and the half width of the imaginary part (black) of the mutual inductance $(T \leq 1 \mathrm{~K})$ confirms the film uniformity. These results (obtained without using a buffer and/or protection layers) are comparable with the results reported by the Bozovic group from the Brookhaven National Laboratory, USA [87]. The high structural quality of the film is confirmed by atomic-resolution STEM-HAADF imaging. The LSCO film (brighter) is distinguished from the LSAO substrate (darker) exhibiting a perfect coherent interface (Fig. 2c).

a

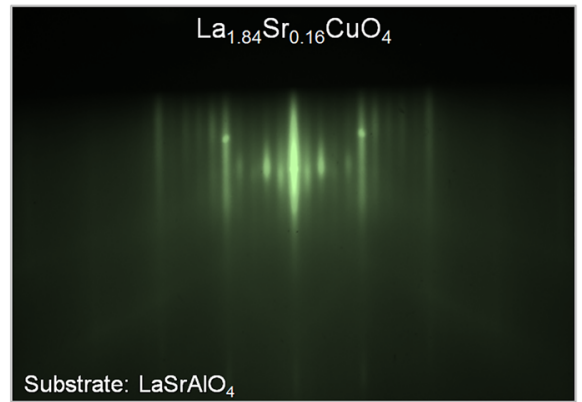

b

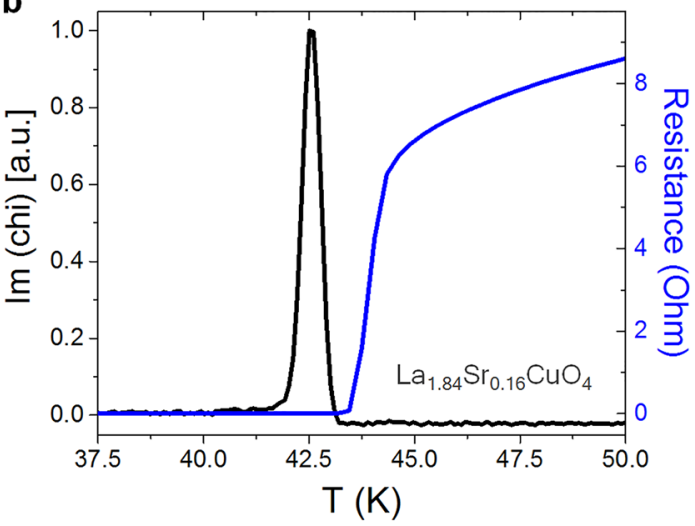

Fig. 2 Characterization of optimum doped $\mathrm{La}_{1.84} \mathrm{Sr}_{0.16} \mathrm{CuO}_{4}$ films. a Representative in situ RHEED image of the optimum-doped LSCO $(x \approx 0.16)$ film acquired after a completed deposition of a LSCO unit cell. The reconstruction pattern corresponds to a $5 \mathrm{a}$ in-plane spacing. $\mathbf{b}$ The imaginary part of the mutual inductance together with the resistance vs. temperature plot of the LSCO $(x \approx 0.16)$ film exhibiting a sharp
High-quality LSCO epitaxial films are synthesized in a vastly oxidized environment, which is achieved via the delivery of pure ozone by evaporation of liquid ozone from a distilled system. The typical substrate temperature is $\sim 650{ }^{\circ} \mathrm{C}$ according to the radiative pyrometer reading and the chamber pressure is $\sim 10^{-5}$ Torr. The highest $T_{c}$ value of the films suggests that most of the oxygen positions in the LSCO crystal structure are occupied by oxygen ions. The undoped LCO thin film grown under these conditions is superconducting with $T_{c}>40 \mathrm{~K}$ due to the intercalation of oxygen interstitials. In the LSCO unit cell, there are three unequal oxygen positions with different binding energies: (i) the oxygen atoms in the $\mathrm{CuO}_{2}$ plane, (ii) the apical oxygen atoms, and (iii) the oxygen interstitial(s). There are different ways to govern the oxygen content such as post-growth annealing in vacuum or oxygen/ ozone [88] and post-growth electrochemical doping [78, 83, 89, 90]. Nevertheless, since the thermogravimetric analysis (TGA) is not applicable for such thin films to evaluate the amount of oxygen due to their small volume, another method should be used to qualitatively verify the oxygen concentration. For this, we measure the electrical conductivity of a single LCO film in the temperature interval $300 \mathrm{~K}<T<$ $800 \mathrm{~K}$ (see Fig. 3) [91]. The decrease of the electrical conductivity at $300 \mathrm{~K}<T<500 \mathrm{~K}$ (region 1) is ascribed to the loss of non-equilibrium interstitial oxygens, which, accordingly,

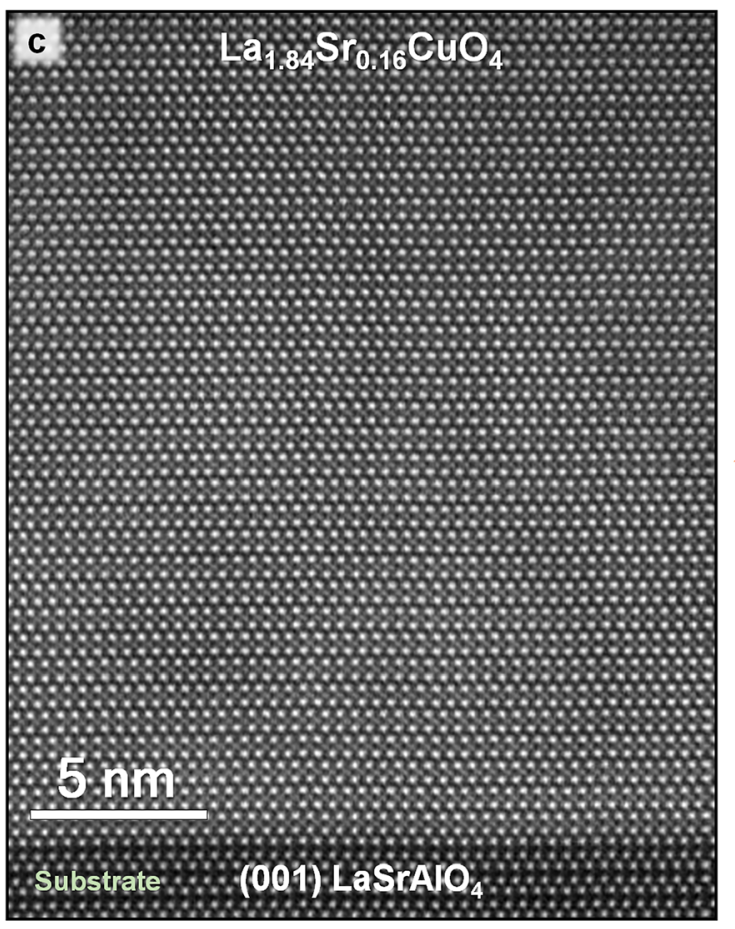

superconducting transition with $T_{c}(R=0) \approx 43 \mathrm{~K}$. c The atomicresolution STEM-HAADF image of the LSCO film grown on a LSAO (001) substrate displays a perfect structural quality, an absence of any extended defects, and a coherent interface between the substrate and the epitaxial film. The orange arrow next to the HAADF image indicates the growth direction 


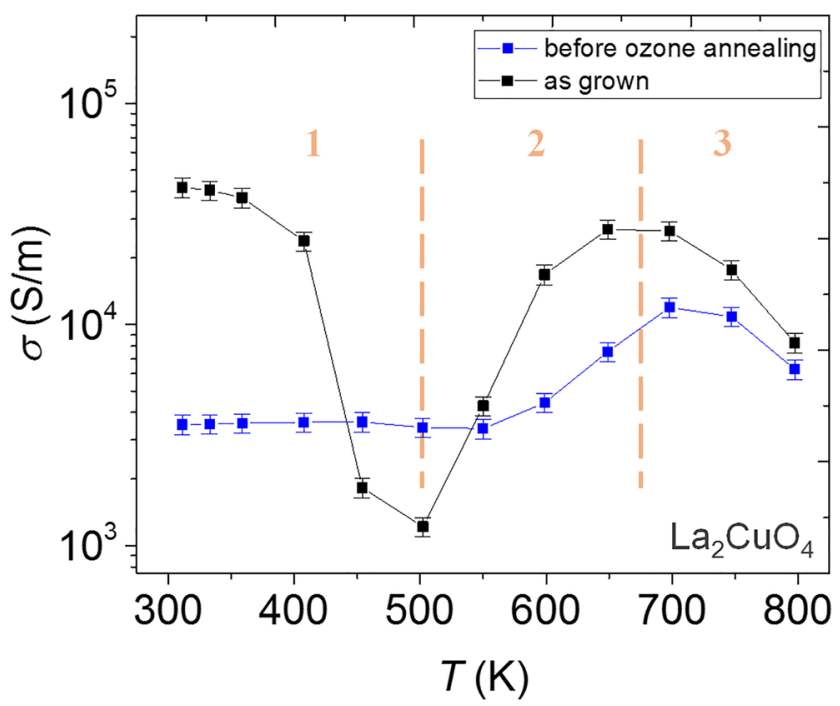

Fig. 3 High-temperature oxygen exchange reaction of undoped $\mathrm{La}_{2} \mathrm{CuO}_{4}$ [91]. The electrical conductivity of a $37 \mathrm{ML}$ LCO is displayed as a function of temperature (i) for the "first heating" (i.e., after the growth; "as grown") and (ii) for a second heating experiment. Orange dashed lines indicate the distinguished temperature regions and " 2 nd heating" refers to the (repeated) measurement performed on the "as grown" sample after the first measurement cycle (heating and cooling) without taking the sample out of the MBE chamber

results in a decrease of the number of holes. Oppositely, in the region 2 (i.e., 500-650 K), the oxygen content in equilibrium increases with increasing temperature similar to what has been reported in Ref. [82], whereas kinetic effects start to disappear as the temperature is increased further (region $3, T>650 \mathrm{~K}$ ). However, in the "colder" region for $T \leq 550 \mathrm{~K}$, the oxygen atoms are quenched, and the system gets stable. Herewith, we show that the oxygen concentration of complex oxides, which have a strong influence on transport properties, can be examined through high-temperature conductivity measurements, as we discussed for undoped LCO films. Since examining the electrical properties above room temperature is a promising way to investigate oxygen exchange dynamics, a possible extension of this method can be conducting the experiments under different oxygen/ozone pressure.

\section{Diverse Epitaxial Design}

Thus far, we have presented high-quality L(S)CO epitaxial films grown by ozone-assisted MBE. Analogously, all other oxide hetero-structures synthesized by our MBE system have similar distinguished characteristics from the point of stoichiometry, crystal structure, and minimal concentration of oxygen defects. Fortunately, with the advantage of atomic-layer precise controlled growth, different deposition schemes can be further constructed utilizing in situ RHEED especially at the distinct layer transitions.
The unit cell of the intrinsically layered LCO structure comprises two molecular layers and each molecular layer is built up of two $\mathrm{LaO}$ and one $\mathrm{CuO}_{2}$ atomic layers. Thus, employing the layer-by-layer deposition scheme, this layered oxide compound can be formed by using either in $\mathrm{La}-\mathrm{La}-\mathrm{Cu}$ or in $\mathrm{Cu}-\mathrm{La}-\mathrm{La}$ deposition sequences. However, the deposition of the metals in oxidation conditions and the layer-bylayer regime lead to the formation of nanoscale metal oxides: $\mathrm{La}_{2} \mathrm{O}_{3}$ and $\mathrm{CuO}$. The 214-LCO structure is a result of the surface chemical reaction between these elementary oxides, which is derived by the electronegativity of the constituent metals. Therefore, the proper deposition sequence is La-La$\mathrm{Cu}$ (see Fig. 4a) instead of $\mathrm{Cu}-\mathrm{La}-\mathrm{La}$, as the $\mathrm{Cu}$ atoms with the largest electronegativity pick up the oxygen from $\mathrm{La}_{2} \mathrm{O}_{3}$ and form $\mathrm{CuO}_{2}$ planes generating the 214 building block. In addition to the alternation of single oxide layers in a single-phase compound, a variety of multilayers, for instance bilayers (Fig. 2b), (or multilayers in the case of more repetitions) or two-dimensional doping (so-called $\delta$-doping), in which a selected single metal oxide atomic layer can be substituted with another metal (i.e., dopant), can be designed as sketched in Fig. 2c.

In the case of multilayer synthesis, one can define the multilayer structure either as homo-epitaxial or as hetero-epitaxial depending on the constituent layers. In case the multilayer is composed of epitaxial layers of the same material with the same crystal structure but different doping levels, the structures are called homo-epitaxial structures. On the other hand, when the multilayer is built up by contacts of different materials, e.g., by stacking of cuprates and nickelates, or cuprates and manganates, or nickelates and manganates, with different chemical formula and crystal structure, the structures are called hetero-epitaxial. Note that for both homo-epitaxy and hetero-epitaxy, the multilayers can be grown on the substrate of different materials, in such a situation the growth of the initial layers is always hetero-epitaxy.

\section{Homo-Epitaxy and HT-IS of $\mathrm{La}_{2} \mathrm{CuO}_{4}$}

A milestone in the field of LCO-based HTS was reached in 2008 by the discovery of interface superconductivity between strongly overdoped metallic (M) LSCO and undoped insulating (I) LCO layers, which are not superconducting when taken alone $[26,94]$. It is also shown that the superconducting transition temperature depends on the layer sequence of the M, I, and superconducting (S) layers. Further details can be found in the reviews devoted to this HT-IS phenomenon $[86,95,96]$ and the original references therein. Herewith, we summarize the main findings and conclusions:

i. I-M bilayers exhibit lower $T_{c} \leq 15-20 \mathrm{~K}$ as $T_{c}$ for M-I and M-S bilayers, where $T_{c} \approx 30-36 \mathrm{~K}$ and $T_{c} \approx 51 \mathrm{~K}$ can be 
a

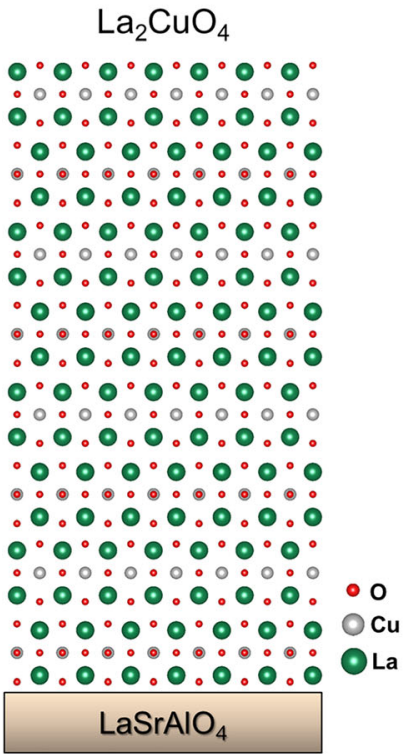

b

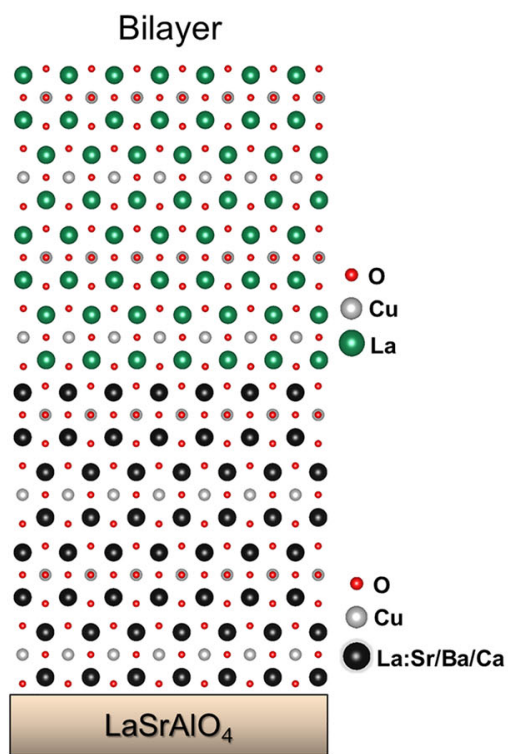

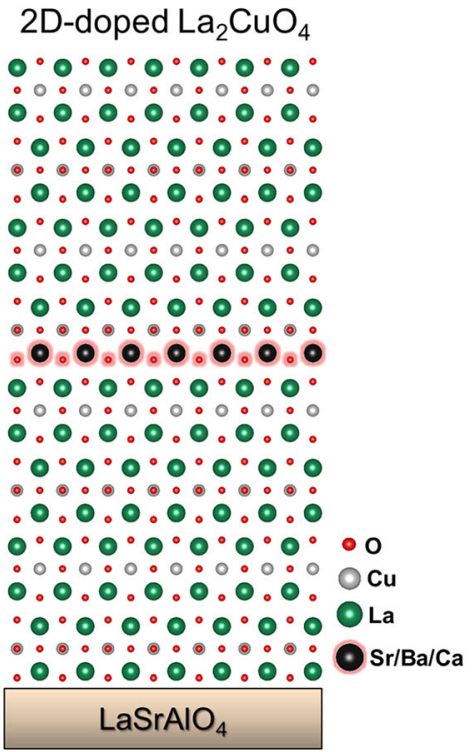

Fig. 4 Different designs of $\mathrm{La}_{2} \mathrm{CuO}_{4}$ homo-epitaxial structures. Basic sketches of a single-phase LCO, b metal (overdoped)-insulator (undoped) bilayer, c 2D-doped LCO grown on LSAO (001) substrates.
The $\mathrm{La}_{2} \mathrm{CuO}_{4}$ structure forms a pseudo-tetragonal structure when grown on $\mathrm{LaSrAlO}_{4}$ substrates [92, 93] achieved, respectively. (Note that $\mathrm{S}$ indicates the superconducting LCO layer after post-annealing in ozone atmosphere).

ii. The thickness of the superconducting interface layer is very thin and confined to a few $\mathrm{CuO}_{2}$ planes. $\mathrm{Zn}$ tomography [94] enabled to pinpoint the exact position of the superconducting $\mathrm{CuO}_{2}$ plane with the highest $T_{c}$.

iii. The possible mechanism due to the nominal $\mathrm{La}-\mathrm{Sr}$ intermixing at the interface has been ruled out by several independent experiments including in situ time-of-flight ion scattering and recoil spectroscopy during growth (TOF-ISARS) and high-resolution STEM [26]. The cation intermixing is confined within one unit cell from the nominal interface as supported by the real-time in situ RHEED observations during the growth.

iv. The importance of the crystal structure in I-M, M-I, and M-S bilayers has been shown by Butko et al. [97] via high-resolution X-ray diffraction studies demonstrating the pseudomorphic growth of the top layer in both M-I and I-M bilayers. Importantly, $T_{\mathrm{c}}$ is linearly dependent on the $c$-axis lattice parameter. The larger the $c$-lattice parameter, the higher $T_{\mathrm{c}}$. A similar relationship between the $c$-lattice parameter and $\mathrm{Cu}-\mathrm{O}$ distances has been also reported for single-phase LSCO films [6, 98], and besides, the Coherent Bragg Rod Analysis (COBRA) method for M-I bilayers revealed another structural effect. An increase of the copper-apical oxygen interatomic distances starting from the nominal M-I interface towards the bilayer surface is substantiated [99, 100]. These structural results have been discussed in a frame of a long-range "Madelung strain" effect ascribed to strong Coulomb interaction between the layers, which is mildly screened in growth direction [97, 101].

v. Finally, a charge transfer mechanism due to the chemical potential difference between the overdoped and undoped cuprate layers has been proposed for HT-IS in M-I, I-M, and M-S bilayers. The phenomenological model (based on the Poisson equation) for the electrostatic potential is employed to determine the charge profile across the interfaces. Taking the doping dependence of the chemical potential $\mu(\mathrm{p})$ for the LSCO compound based on angleresolved photoemission spectroscopy (ARPES) results [102], the anomalous independence of $T_{c}$ for HT-IS on the doping level of the M layer in M-I bilayers was supported [103]. The open question is the $\mu(\mathrm{p})$ independency in the pseudogap regime (from undoped to optimum doping level), while $\mu$ is linearly dependent in the overdoped regime. Several mechanisms have been proposed (e.g., Ref. [104]); however, this is out of the scope of this review.

In addition to above-mentioned research, we further utilized the advantages of the layer-by-layer MBE technique. Bauitti et al. [105] reported a new approach for the design of epitaxially grown HTS of cuprates and showed that when twodimensional doping, i.e., $\delta$-doping, which is the substitution of a selected La-O layer with a Sr-O layer during the epitaxial growth, is applied to the LCO matrix, an asymmetric 
distribution of the dopant is present $[105,106]$. For such type of doping, the asymmetric dopant distribution profile generates two different types of HTS mechanisms at each side of the interface. While in the growth direction, where the Sr distribution is wide and accompanies to the hole distribution, "conventional doping" is the main mechanism. On the other side of the interface, which has found to be sharper, the dopant, i.e., $\mathrm{Sr}$, and the charge carrier distribution profiles are decoupled, and such decoupling results in a "space-charge" region inducing HTS. Further details can be found in Ref. [105]. The effect of the cation size and the growth temperature on this asymmetric profile has been further studied with atomic-resolution STEM for analogous systems [107], substantiating the fact that although the cation size mildly affects the dopant distribution width in both growth and substrate directions, the asymmetric dopant distribution profile is present and independent of the cation size.

Since the importance of the crystal structure has been demonstrated for HT-IS, the chemical pressure based on the dopant size is expected to perturb the crystal structure and correspondently to modify electronic and superconducting properties. Dopant size effects on the HT-IS were studied by Suyolcu et al. [76], and it has been demonstrated that although HTS can be obtained (Fig. 5a) by employing dopants with different sizes but same valences (i.e., $\mathrm{Sr}^{2+}, \mathrm{Ba}^{2+}$, and $\mathrm{Ca}^{2+}$ ), depending on the interface structure, both "interfacial" and "conventional" HTS can be realized. In particular, although sharp interfaces were achieved via $\mathrm{Ca}$ and $\mathrm{Sr}$ doping (in M layer) that confined the HTS into 1-2-nm scale, due to the strong surface segregation of Ba (first observed during the growth, Fig. 5b) for Ba-doped M-I bilayers, the HT-IS is hindered as a consequence of the randomly distributed dopants within the entire sample. For the latter case, the bilayer with high epitaxial quality (Fig. $5 \mathrm{c}$ ) turns into a conventionally doped LCO system and most of the layers were found to be superconducting. Furthermore, when the local octahedral distortions, i.e., apical O-O interatomic distances, are quantitatively investigated and associated with the dopant distributions, the correlation with local Jahn-Teller distortions is enlightened [93]. While in the case of sharp interfaces (i.e., $\mathrm{Ca}$ and $\mathrm{Sr}$ doping), the O-O interatomic distances increase sharply after the nominal M-I interface, anti-JahnTeller distortions dominates the structure for Ba doping due to the wide $\mathrm{Ba}$ distribution leading to a reduction of the apical O-O interatomic distances [109]. As an example, a superimposed image of simultaneously acquired STEM high-angle annular dark-field (HAADF) and STEM-ABF images is displayed in Fig. 5d. These recent studies, including the 2D-doped LCO structure and a comparative study of dopant size effects on HT-IS, demonstrated the significant role of intermixing at interfaces, which was neglected in previous studies: The interfacial intermixing has an impact on the local crystal structure and, correspondingly, on the physical properties.

\section{Hetero-Epitaxy of $\mathrm{La}_{2} \mathrm{CuO}_{4}$}

In addition to homo-epitaxial oxide systems, which concern the doping dependency of different physical properties, hetero-epitaxial systems provide a fundamental playground for device fabrication and possible applications. Certainly, to fabricate devices $[110,111]$, it is a prerequisite to combine several materials, e.g., superlattices, in which apprehending the hetero-epitaxial interfaces is momentous to interpret and improve the underlying structural and physical properties. Hence, one needs to zoom in on the interfaces, not only because of the fact that complex oxides are characterized by ultra-short characteristic length scales such as the Debye screening length or the superconducting coherence length, but also the nanoscopic distortions at interfaces can profoundly affect device properties.

One recent example of hetero-interface engineering by oxide MBE is the HT-IS with a critical temperature up to $T_{c} \approx$ $40 \mathrm{~K}$ at $\mathrm{LCO}-\mathrm{La}_{2-x} \mathrm{Sr}_{x} \mathrm{NiO}_{4}$ (LSNO) multilayers (Fig. 6a) [112]. Note that LCO and LSNO compounds share the same Ruddlesden-Popper 214-crystal structure, and the electronic phase transformation can be achieved by hole-carriers doping by the substitution of the La site with a Sr dopant. It is revealed that $T_{c}$ of such LCO-LSNO hetero-structures can be tuned by changing the doping level $x$ in the LSNO layer or by varying the LCO thickness. The conditions to emerge HT-IS are the following: The critical doping level in the LSNO layer for a constant LCO layer thickness, $N=2.5$, is $x>0.5$ and the critical LCO thickness for constant $x=1.3$ is $N \geq 1.5$ unit cells, while the highest $T_{c}>30 \mathrm{~K}$ was obtained for $2.5<N<4.5$, with $N$ as the number of unit cells in the crystallographic $c$ direction. For larger $N$ corresponding to larger thicknesses, $T_{c}$ saturates at around $25 \mathrm{~K}$. Both interfaces, i.e., LCO-LSNO and LSNO-LCO, have been examined by aberrationcorrected STEM and the structurally coherent interfaces are substantiated as presented in Fig. 6d, whereas STEM-EELS investigations revealed a certain redistribution of both A-site $(\mathrm{La}, \mathrm{Sr})$ and $\mathrm{B}$-site $(\mathrm{Cu}, \mathrm{Ni})$ cations. In particular, although the LCO (bottom)-LSNO (top) interface is found to be sharp, an obvious $\mathrm{Sr}$ redistribution in the LCO phase in growth direction is detected, which slightly changes depending on the doping level in the LSNO layer. The details are discussed in Ref [112]. Lastly, the broad resistance transition to the superconducting state at such high-onset temperature (similar to optimum-doped LSCO) and the reduced Meissner effect should also be pointed out, since this is an indirect evidence of the inhomogeneous superconducting interface with nanoscale superconducting puddles as a consequence of the interface intermixing. 
Fig. 5 Chemical pressure effects on M-I bilayers [76, 93]. a Transport measurements reveal that all samples, i.e., Sr- (green), $\mathrm{Ba}$ - (red), and Ca-doped (blue) M-I bilayers exhibit HTS. b RHEED pattern obtained from a LBCO-LCO bilayer indicates secondary phase formation realized by additional diffraction spots. The white arrows indicate some of the additional diffraction spots. c The low-magnification STEM-HAADF image taken from the LBCO-LCO bilayer reveals the long-range structural quality of the sample. d

Superposition of simultaneously acquired STEM-HAADF and STEM-ABF images visualizing all atomic columns along this projection, from which the interatomic $\mathrm{O}-\mathrm{O}$ distances are quantitatively measured [108]. The orange arrows in $\mathbf{c}$ and $\mathbf{d}$ indicate the growth direction and the yellow arrows in $\mathbf{d}$ mark the LSAO substrate-overdoped LBCO and overdoped LBCOundoped LCO interfaces a
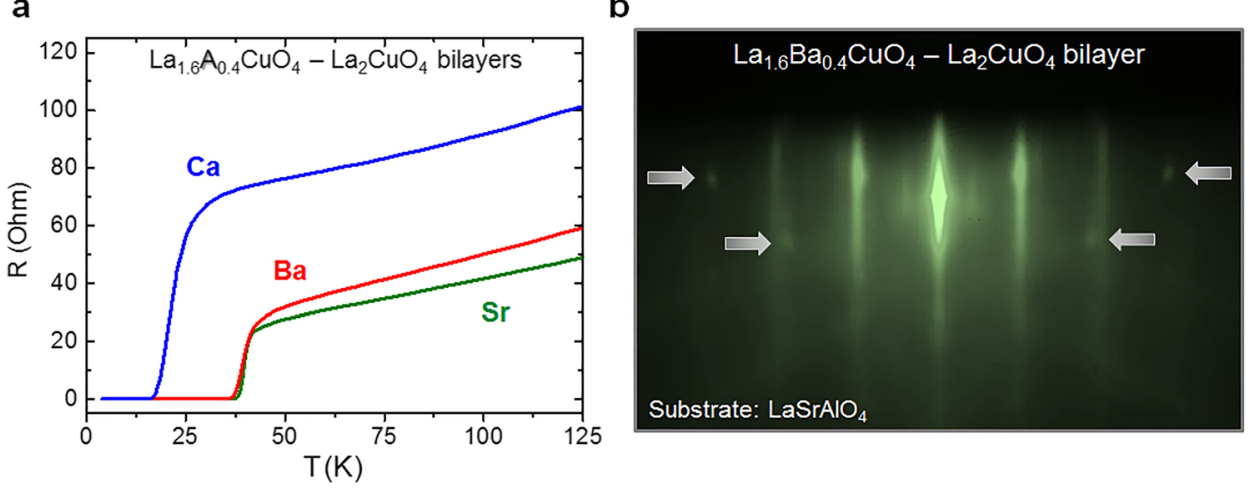

C

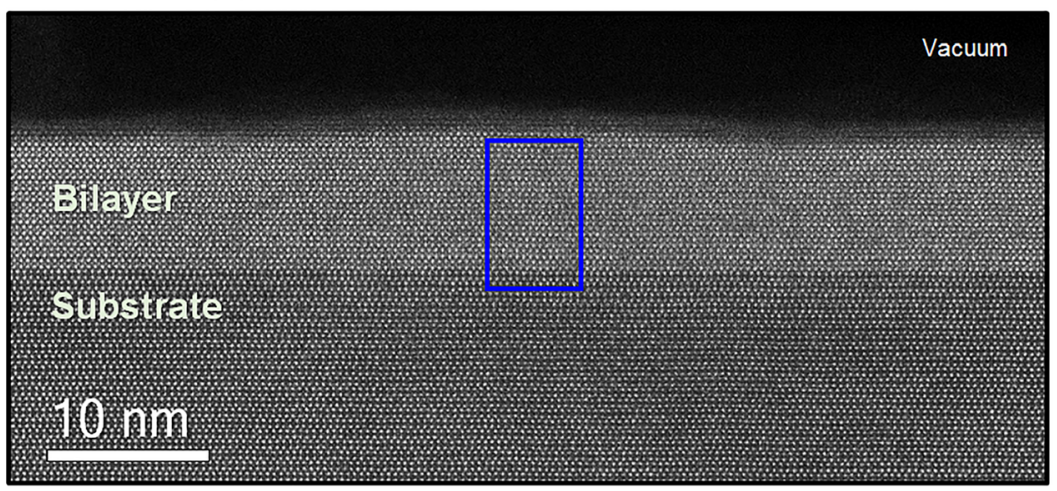

d

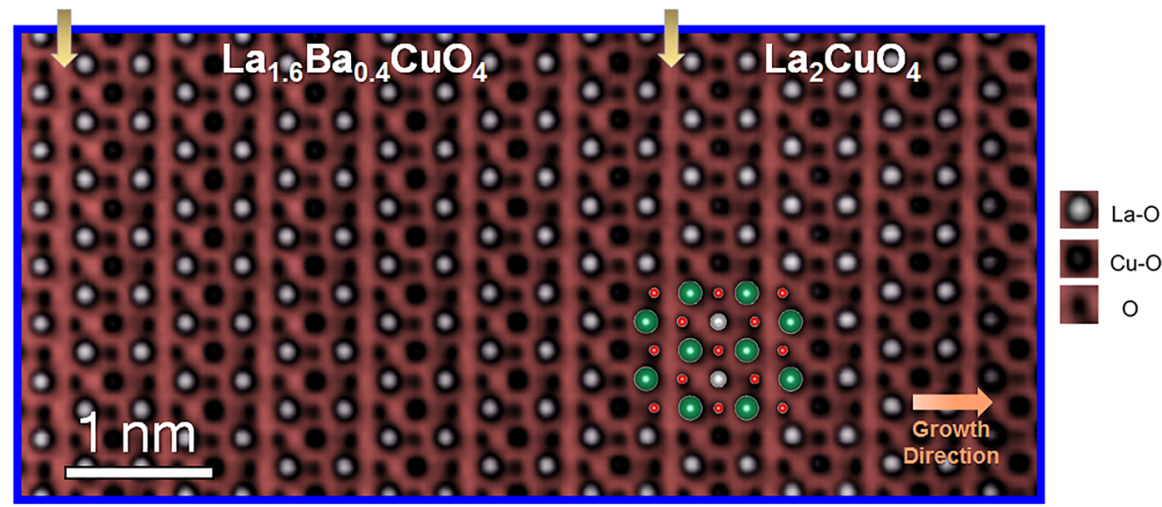

As discussed earlier in the text, different parameters have a strong impact on the interfacial structure, and an intermixing layer with a thickness of 1-2 unit cells is present at the interfaces of the 214-214 structures. Interfaces of oxide layers with different crystal structures, e.g., 214 pseudo-tetragonal and 113 cubic-perovskite, can be further designed. An intriguing example with contacts of insulating 214-LCO and metallic 113- $\mathrm{LaNiO}_{3}$ (113-LNO) multilayers (Fig. 6b) has been recently studied for examining the thermoelectric and transport properties [91]. The dependency of the transport properties on the thickness of the constituent layers was revealed. As the individual layers' thicknesses are reduced, the Seebeck coefficient changes the sign and the electrical conductivity is decreased. High-resolution STEM imaging substantiated that a solid solution of $\mathrm{La}_{2}(\mathrm{Cu}, \mathrm{Ni}) \mathrm{O}_{4}$ with 214 crystal structure forms, when the thickness of the 113-LNO layer is decreased down to 2 unit cell. This length scale is comparable with the critical length of elemental intermixing, while for the thick LNO layer, the structural (Fig. 6e) and also the chemical abruptness of the interfaces has been visualized, when the nominal LNO layer thickness is locked to eight unit cells [91].

Another prominent example of 214 and 113 crystal structure junctions is the interface between superconducting LSCO and ferromagnetic manganite $\mathrm{La}_{0.66} \mathrm{Sr}_{0.33} \mathrm{MnO}_{3}$ (LSMO) layers [114]. Here, we focus on cuprate-manganate heterostructures (Fig. 6c) and present individual superconducting $(\mathrm{S})$ cuprate and ferromagnetic $(\mathrm{F})$ manganate hetero-interfaces. Due to the incompatibility of ferromagnetism and superconductivity, this interface is a playground to study unconventional proximity effects [115, 116]. For similar, i.e., cuprate-manganate, oxide heterostructures, the antiferromagnetic coupling driven by orbital reconstruction and charge transfer emerging at the interface has been reported [117]. The main challenge of such design 
214 cuprate -214 nickelate

a

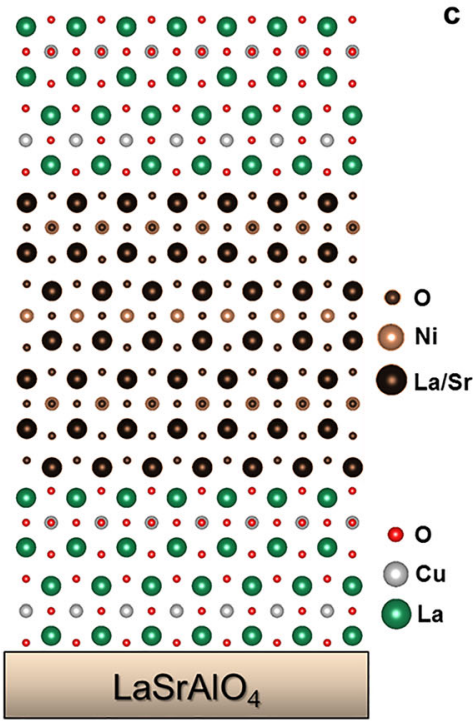

b

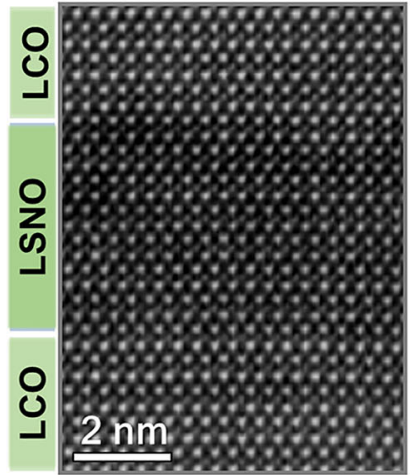

214 cuprate -113 nickelate

c

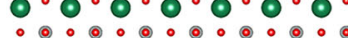

०.०.०.०.०.०.० 。

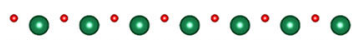

0.000 .0 .0 .0 .00

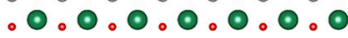

- $0^{\circ} 0^{\circ} 00^{\circ} \circ 0^{\circ} 0$

$\bullet \bullet \circ \odot \bullet \bullet \odot \circ \odot \circ \odot \circ \odot$

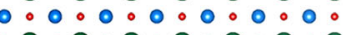

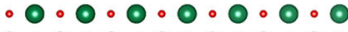

$0 \circ 0 \circ 0 \circ 0.000000$

.0 .0 .0 .0 .0 .0 .0 .0

$0.000 .0 \circ 0.0 \circ 0.0 \mathrm{Ni}$

$\circ .0000000$

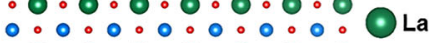

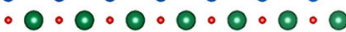

0.000 .000 .000$.

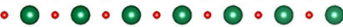

$0^{\circ} 0^{\circ} 0^{\circ} 0^{\circ} \odot 0^{\circ} \odot 0^{\circ}$

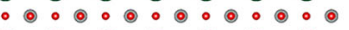

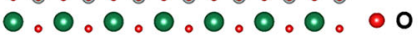

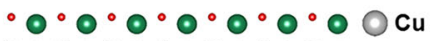

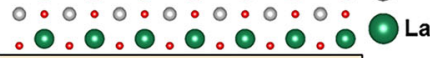

$\mathrm{LaSrAlO}_{4}$

d

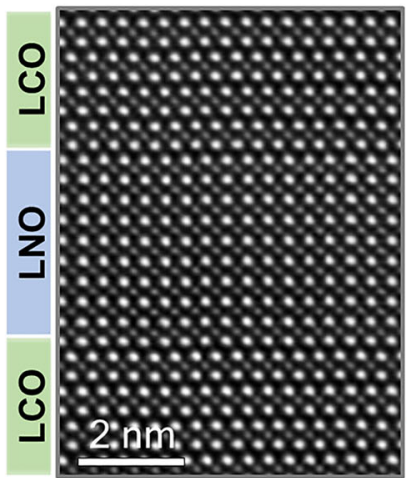

214 cuprate - 113 manganite

e

( $0^{\circ} 0^{\circ} 0^{\circ} 0^{\circ} 0^{\circ} 0^{\circ}$

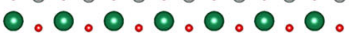

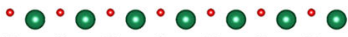

0.000 .000 .0000

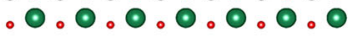

$0_{0}^{\circ} 0^{\circ} 0^{\circ} 0^{\circ} 0^{\circ} 0^{\circ} 0^{\circ}$

$\circ \odot \circ \circ \odot \circ \odot \circ \odot \circ \odot \circ \odot$

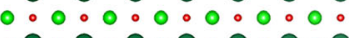

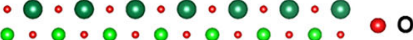

$\bullet \bullet \circ \bullet \bullet \circ \odot \circ \odot \circ \odot \circ \odot \bigcirc \mathrm{Mn}$

(1)

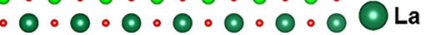

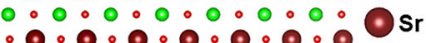

0.000 .0000000

$\circ \bullet \bullet \circ \bullet \circ \odot \circ \odot \circ \bullet \circ \odot$

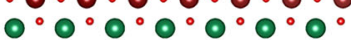

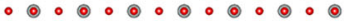

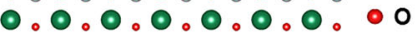

$\circ 0^{\circ} \odot^{\circ} \odot^{\circ} \odot^{\circ} 0^{\circ} \odot$ OCu

$\circ: \circ \circ \circ \circ \circ \circ \circ: 0$ La

$\mathrm{LaSrAlO}_{4}$

f

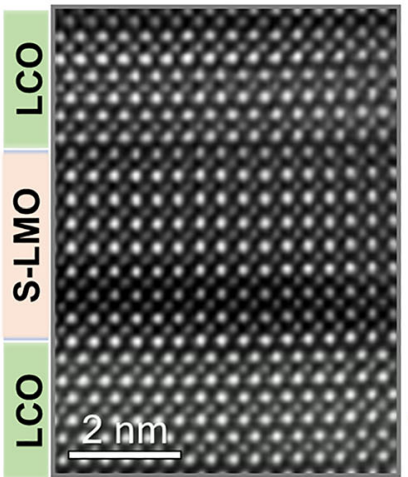

Fig. 6 Different hetero-epitaxial designs. Basic sketches of a LCOLSNO-LCO trilayer [Ref. [112]], c LCO-LNO-LCO multilayer [Ref. [91]], e LCO-SMO-LMO-LCO multilayer [Ref. [113]] grown on (001) LSAO substrates. Atomic-resolution STEM-HAADF images of b LCO-
LSNO-LCO interfaces and $\mathbf{d}$ LCO-LNO-LCO interfaces and $\mathbf{f}$ LCOSMO-LMO-LCO showing the structural coherency between the layers. The pink arrows indicate the growth direction for the different multilayers is preserving both superconducting and ferromagnetic properties in the multilayers with an appropriate interface quality. Recently, it was shown that the perpendicular exchange bias in $\mathrm{L}(\mathrm{S}) \mathrm{CO}-\mathrm{L}(\mathrm{S}) \mathrm{MO}$ hetero-structures is caused by the antiferromagnetic interaction between copper and manganese ions at the interface. The magnitude of the perpendicular exchange bias was systematically tuned by adjusting the doping level of cuprate layers [113], where all the superlattices were prepared with analogue ferromagnetic layers (i.e., $2 \times \mathrm{SrMnO}_{3}(\mathrm{SMO})+4 \times \mathrm{LaMnO}_{3}(\mathrm{LMO})$ layers) $[118,119]$. Additionally, the doping level of LCO layers was varied utilizing the atomic layer-by-layer MBE in order to point the charge transfer scenario. To illustrate the quality of the cuprate-manganate interfaces, a representative high-resolution STEM-HAADF image is presented in Fig. 6f. The sharp and structurally coherent interface between 214 pseudo-tetragonal [92] and 113 cubic structures is discernible. The darker contrast on the image corresponds to the SMO layer due to the weaker Z-contrast stemming from lighter Sr ions compared with the heavier La. The chemical intermixing and physical properties of these interfaces will be published elsewhere.

\section{Concluding Remarks and Outlook}

During the last decade, significant progress has been achieved in the field of complex oxide hetero-structures, albeit there is still a huge room to explore. Triggered by the continuous development of the atomically precise deposition technologies, the quality of not only the oxide thin films but also the interfaces has improved drastically. In this review, we touch on several examples of epitaxial design including HTS at the interfaces of overdoped and undoped LCO layers, Sr deltadoped LCO structures and heavily Sr-doped LSNO and undoped LCO layers and the realization of abrupt interfaces 
between different crystal structures, e.g., tetragonal 214 and cubic perovskite 113, are presented.

Utilizing the ozone-assisted layer-by-layer oxide MBE synthesis, one can manipulate the layer sequences with atomic-layer precision and also tune the interface properties, correlatively. Aberration-corrected STEM with atomicresolution imaging and spectroscopic tools allow the identification of individual atomic columns in the synthesized structures: The crystal structure across the oxide interfaces, where the novel phenomena emerge, is structurally coherent and well defined, while the chemical intermixing is present in most of the cases. Taking into account that the intrinsic physical properties of the complex oxides are a result of the combination of the crystal structure symmetry and doping, the observation of perfect interfaces with confined dopant distribution profiles rises a fundamental question on whether the interface properties are the consequences of only doping profiles or that some other non-trivial mechanisms are involved as well. Independent on the answer of this query, we do have the technologies to fabricate complex oxide hetero-structures with different functional properties that can be used in future applications. Nevertheless, the acceptance of this fundamental question and the accurate answer can provide a further understanding of the physics of complex oxides as well as the impact of the local chemistry and crystal structure distortion. Finally, yet importantly, in spite of the significant progress in thin film technologies and characterization methods, one can see that the limits of the characterization methods still do not allow us to answer this question clearly. Thereby, we believe that further technical improvements and the better integration of high-end characterization techniques to the growth tools will pave the way for more significant scientific achievements.

\subsection{Experimental}

\subsubsection{Growth of the Samples}

The LCO-based films were grown in an ozone-assisted MBE system (DCA Instruments) in the layer-by-layer regime, in which the sequence of the evaporation of the constituent layers follows the crystal structure of 214 compounds. In a nominal growth, first, two La-O atomic layers are deposited, and this is followed by the deposition of one $\mathrm{Cu}-\mathrm{O}$ atomic layer in accordance with the chemical formula of $\mathrm{La}_{2} \mathrm{CuO}_{4}$. In the case of Sr-doped LCO compounds, a part of La atoms is replaced by $\mathrm{Sr}$ atoms in the co-deposition regime, i.e., simultaneous evaporation of $\mathrm{La}$ and $\mathrm{Sr}$ atoms is in a proportion corresponding to a doping level. The typical time for formation of one molecular layer of $\mathrm{L}(\mathrm{S}) \mathrm{CO}$ was $\sim 3 \mathrm{~min}$. The substrate temperature was measured by the infrared pyrometer and was kept around $650{ }^{\circ} \mathrm{C}$. The total background pressure (ozone $\left(\mathrm{O}_{3}\right)$, molecular oxygen $\left(\mathrm{O}_{2}\right)$, and atomic oxygen $(\mathrm{O})$ ) was $\sim 1.5 \times 10^{-5}$ Torr provided by continuous evaporation of the distilled ozone.

In-Situ Characterization An electron beam emanated from the RHEED gun, which is operated at $25 \mathrm{kV}$, irradiates the substrate surface at an incident angle of $1-2^{\circ}$. The dynamics of the RHEED patterns and the intensities of the diffraction streaks were monitored with a CCD camera and analyzed by using $\mathrm{k}$-Space 400 software.

Structural Characterization and Transport Measurements Surface morphology, crystal structure characterizations, and AES investigations (at $10 \mathrm{kV}$ ) were performed by atomic force microscopy (AFM) (Nanoscope III), high-resolution X-ray diffraction (XRD) (Bruker D8 Cu-K $\alpha 1=1.5406 \AA$ ), and Auger microprobe (JEOL JAMP-7810), respectively. Electrical measurements in a Van der Pauw four-point-probe configuration with alternative DC currents of $\pm 20 \mu \mathrm{A}$ were employed. Simultaneously, measurements of the imaginary and real parts of the mutual inductance $M(T)$ were carried out by magnetic susceptibility measurements in a two-coil configuration in parallel geometry with an $\mathrm{AC}$ current of $50 \mu \mathrm{A}$ at a frequency of $1 \mathrm{kHz}$. The temperature was varied from room temperature to $4.2 \mathrm{~K}$ (liquid helium) using a motorized custom-designed dipstick ( $T$ change rate $<0.1 \mathrm{~K} / \mathrm{s}$ ).

Scanning Transmission Electron Microscopy For representative cross-sectional electron transparent specimens, conventional specimen preparation procedures including mechanical thinning (grinding), tripod wedge polishing, and argonion thinning (with a liquid nitrogen-cooled stage; PIPS II, Model 695) were performed. For all STEM analyses, a probe-aberration-corrected JEOL JEM-ARM200F STEM equipped with a cold field emission electron source, a probe $C_{\mathrm{s}}$-corrector (DCOR, CEOS GmbH), and a Gatan GIF Quantum ERS spectrometer was used. STEM imaging and electron energy loss spectroscopy (EELS) analyses were performed at probe semi-convergence angles of $20 \mathrm{mrad}$ and $28 \mathrm{mrad}$, resulting in probe sizes of $0.8 \AA$ and $1.0 \AA$, respectively. The collection angles for STEM-HAADF and STEMABF images were 75-310 and 11-23 mrad, respectively. A collection semi-angle of $111 \mathrm{mrad}$ was used for EELS investigations [56]. For STEM data process and analyses, different software tools [120-123] have been used.

Acknowledgments Authors gratefully acknowledge Peter Specht for the technical support on the oxide MBE system and Pinar Kaya and Gideok Kim for fruitful discussions.

Funding Information Open access funding provided by Max Planck Society. This project has received funding from the European Union's Horizon 2020 research and innovation program under grant agreement No. 823717-ESTEEM3. 
Open Access This article is distributed under the terms of the Creative Commons Attribution 4.0 International License (http:// creativecommons.org/licenses/by/4.0/), which permits unrestricted use, distribution, and reproduction in any medium, provided you give appropriate credit to the original author(s) and the source, provide a link to the Creative Commons license, and indicate if changes were made.

\section{References}

1. Dagotto, E.: Complexity in strongly correlated electronic systems. Science. 309, 257-262 (2005). https://doi.org/10.1126/science. 1107559

2. Zubko, P., Gariglio, S., Gabay, M., Ghosez, P., Triscone, J.-M.: Interface physics in complex oxide heterostructures. Ann Rev Condens Matter Phys. 2, 141-165 (2011). https://doi.org/10. 1146/annurev-conmatphys-062910-140445

3. Goodenough, J.B., Zhou, J.-S.: Localized to itinerant electronic transitions in transition-metal oxides with the perovskite structure. Chem. Mater. 10, 2980-2993 (1998). https://doi.org/10.1021/ cm980276u

4. Bednorz, J.G., Müller, K.A.: Possible high $T_{c}$ superconductivity in the $\mathrm{Ba}-\mathrm{La}-\mathrm{Cu}-\mathrm{O}$ system. Z Phys B - Condens Matter. 64, 189193 (1986). https://doi.org/10.1007/BF01303701

5. Naito, M., Sato, H., Yamamoto, H.: MBE growth of $(\mathrm{La}, \mathrm{Sr})_{2} \mathrm{CuO}_{4}$ and $(\mathrm{Nd}, \mathrm{Ce})_{2} \mathrm{CuO}_{4}$ thin films. Physica C. 293, 36-43 (1997). https://doi.org/10.1016/S0921-4534(97)01510-4

6. Locquet, J.-P., Perret, J., Fompeyrine, J., Mächler, E., Seo, J.W., Van Tendeloo, G.: Doubling the critical temperature of $\mathrm{La}_{1.9} \mathrm{Sr}_{0.1} \mathrm{CuO}_{4}$ using epitaxial strain. Nature. 394, 453-456 (1998). https://doi.org/10.1038/28810

7. Oh, B., Naito, M., Arnason, S., Rosenthal, P., Barton, R., Beasley, M.R., et al.: Critical current densities and transport in superconducting $\mathrm{YBa}_{2} \mathrm{Cu}_{3} \mathrm{O}_{7-\delta}$ films made by electron beam coevaporation. Appl. Phys. Lett. 51, 852-854 (1987). https://doi. org/10.1063/1.98834

8. Hellman, E.S., Schlom, D.G., Missert, N., Char Jr., K., Harris, J.S., Beasley, M.R., et al.: Molecular-beam epitaxy and deposition of high-Tc superconductors. J. Vac. Sci. Technol., B: Microelectron. Process. Phenom. 6, 799 (1998). https://doi.org/10.1116/1. 584334

9. Locquet, J., Catana, A., Mächler, E., Gerber, C., Bednorz, J.G.: Block-by-block deposition: a new growth method for complex oxide thin films. Appl. Phys. Lett. 64, 372-374 (1994). https:// doi.org/10.1063/1.111151

10. Schlom, D.G., Eckstein, J.N., Hellman, E.S., Streiffer, S.K., Harris, J.S., Beasley, M.R., et al.: Molecular beam epitaxy of layered Dy-Ba-Cu-O compounds. Appl. Phys. Lett. 53, 1660 1662 (1988). https://doi.org/10.1063/1.100443

11. Schlom, D.G.: Molecular beam epitaxial growth of layered Bi-SrCa-Cu-O compounds. J. Cryst. Growth. 102, 361-375 (1990). https://doi.org/10.1016/0022-0248(90)90393-Y

12. Bhattacharya, A., May, S.J.: Magnetic oxide heterostructures. Annu. Rev. Mater. Res. 44, 65-90 (2014). https://doi.org/10. 1146/annurev-matsci-070813-113447

13. Middey, S., Chakhalian, J., Mahadevan, P., Freeland, J.W., Millis, A.J., Sarma, D.D.: Physics of ultrathin films and heterostructures of rare-earth nickelates. Annu. Rev. Mater. Res. 46, 305-334 (2016). https://doi.org/10.1146/annurev-matsci-070115-032057

14. Sulpizio, J.A., Ilani, S., Irvin, P., Levy, J.: Nanoscale phenomena in oxide heterostructures. Annu. Rev. Mater. Res. 44, 117-149 (2014). https://doi.org/10.1146/annurev-matsci-070813-113437
15. Mannhart, J., Schlom, D.G.: Oxide interface - an opportunity for electronics. Science. 327, 1607-1611 (2010). https://doi.org/10. 1126/science. 1181862

16. Hwang, H.Y., Iwasa, Y., Kawasaki, M., Keimer, B., Nagaosa, N., Tokura, Y.: Emergent phenomena at oxide interfaces. Nat. Mater. 11, 103-113 (2012). https://doi.org/10.1038/nmat3223

17. Ramesh, R., Schlom, D.G.: Creating emergent phenomena in oxide superlattices. Nat. Rev. Mater. 1, (2019). https://doi.org/10. 1038/s41578-019-0095-2

18. Ahn, C.H., Rabe, K.M., Triscone, J.-M.: Ferroelectricity at the nanoscale: local polarization in oxide thin films and heterostructures. Science. 303, 488-491 (2004). https://doi.org/ 10.1126/science. 1092508 .

19. Bousquet, E., Dawber, M., Stucki, N., Lichtensteiger, C., Hermet, P., Gariglio, S., et al.: Improper ferroelectricity in perovskite oxide artificial superlattices. Nature. 452, 732-736 (2008). https://doi. org/10.1038/nature06817

20. Ohtomo, A., Hwang, H.Y.: A high-mobility electron gas at the $\mathrm{LaAlO}_{3} / \mathrm{SrTiO}_{3}$ heterointerface. Nature. 427, 423-426 (2004). https://doi.org/10.1038/nature02308

21. Ohta, H., Kim, S., Mune, Y., Mizoguchi, T., Nomura, K., Ohta, S., et al.: Giant thermoelectric Seebeck coefficient of a twodimensional electron gas in $\mathrm{SrTiO}_{3}$. Nat. Mater. 6, 129-134 (2007). https://doi.org/10.1038/nmat1821

22. Hepting, M., Green, R.J., Zhong, Z., Bluschke, M., Suyolcu, Y.E., Macke, S., et al.: Complex magnetic order in nickelate slabs. Nat. Phys. 1, 1097 (2018). https://doi.org/10.1038/s41567-018-0218-5

23. Tokura, Y., Tomioka, Y.: Colossal magnetoresistive manganites. J. Magn. Magn. Mater. 200, 1-23 (1999). https://doi.org/10.1016/ S0304-8853(99)00352-2

24. Brinkman, A., Huijben, M., van Zalk, M., Huijben, J., Zeitler, U., Maan, J.C., et al.: Magnetic effects at the interface between nonmagnetic oxides. Nat. Mater. 6, 493-496 (2007). https://doi.org/ 10.1038/nmat1931

25. Reyren, N., Thiel, S., Caviglia, A.D., Kourkoutis, L.F., Hammerl, G., Richter, C., et al.: Superconducting interfaces between insulating oxides. Science. 317, 1196-1199 (2007). https://doi.org/10. 1126/science. 1146006

26. Gozar, A., Logvenov, G., Kourkoutis, L.F., Bollinger, A.T., Giannuzzi, L.A., Muller, D.A., et al.: High-temperature interface superconductivity between metallic and insulating copper oxides. Nature. 455, 782-785 (2008). https://doi.org/10.1038/ nature 07293

27. Dijkkamp, D., Venkatesan, T., Wu, X.D., Shaheen, S.A., Jisrawi, N., Min-Lee, Y.H., et al.: Preparation of Y-Ba-Cu oxide superconductor thin films using pulsed laser evaporation from high Tc bulk material. Appl. Phys. Lett. 51, 619-621 (1987). https://doi.org/10. 1063/1.98366

28. Wu, X.D., Inam, A., Venkatesan, T., Chang, C.C., Chase, E.W., Barboux, P., et al.: Low-temperature preparation of high $T_{c}$ superconducting thin films. Appl. Phys. Lett. 52, 754-756 (1988). https://doi.org/10.1063/1.99337

29. Koster, G., Rijnders, G.J.H.M., Blank, D.H.A., Rogalla, H.: Imposed layer-by-layer growth by pulsed laser interval deposition. Appl. Phys. Lett. 74, 3729-3731 (1999). https://doi.org/10.1063/ 1.123235

30. Choi, E.-M., Bernardo, A.D., Zhu, B., Lu, P., Alpern, H., Zhang, K.H.L., et al.: 3D strain-induced superconductivity in $\mathrm{La}_{2} \mathrm{CuO}_{4+\delta}$ using a simple vertically aligned nanocomposite approach. Sci. Adv. 5, eaav5532 (2019). https://doi.org/10.1126/sciadv.aav5532

31. Das, S., Tang, Y.L., Hong, Z., Gonçalves, M.A.P., McCarter, M.R., Klewe, C., et al.: Observation of room-temperature polar skyrmions. Nature. 568, 368 (2019). https://doi.org/10.1038/ s41586-019-1092-8

32. Monkman, E.J., Adamo, C., Mundy, J.A., Shai, D.E., Harter, J.W., Shen, D., et al.: Quantum many-body interactions in digital oxide 
superlattices. Nat. Mater. 11, 855-859 (2012). https://doi.org/10. 1038/nmat3405

33. King, P.D.C., Wei, H.I., Nie, Y.F., Uchida, M., Adamo, C., Zhu, S., et al.: Atomic-scale control of competing electronic phases in ultrathin $\mathrm{LaNiO}_{3}$. Nat. Nanotechnol. 9, 443-447 (2014). https:// doi.org/10.1038/nnano.2014.59

34. Nair, H.P., Liu, Y., Ruf, J.P., Schreiber, N.J., Shang, S.-L., Baek, D.J., et al.: Synthesis science of $\mathrm{SrRuO}_{3}$ and $\mathrm{CaRuO}_{3}$ epitaxial films with high residual resistivity ratios. APL Mater. 6, 046101 (2018). https://doi.org/10.1063/1.5023477

35. Mundy, J.A., Brooks, C.M., Holtz, M.E., Moyer, J.A., Das, H., Rébola, A.F., et al.: Atomically engineered ferroic layers yield a room-temperature magnetoelectric multiferroic. Nature. 537, 523527 (2016). https://doi.org/10.1038/nature19343

36. Nie, Y.F., Zhu, Y., Lee, C.-H., Kourkoutis, L.F., Mundy, J.A., Junquera, J., et al.: Atomically precise interfaces from nonstoichiometric deposition. Nat. Commun. 5, 4530 (2014). https:// doi.org/10.1038/ncomms5530

37. Ivashko, O., Horio, M., Wan, W., Christensen, N.B., McNally, D.E., Paris, E., et al.: Strain-engineering Mott-insulating $\mathrm{La}_{2} \mathrm{CuO}_{4}$. Nat. Commun. 10, 786 (2019). https://doi.org/10. 1038/s41467-019-08664-6

38. Baiutti, F., Wrobel, F., Christiani, G., Logvenov, G.: 3 - Oxide molecular beam epitaxy of complex oxide heterointerfaces. In: Pryds, N., Esposito, V. (eds.) Metal Oxide-Based Thin Film Structures, pp. 53-78. Elsevier (2018). https://doi.org/10.1016/ B978-0-12-811166-6.00003-0.

39. Wrobel, F., Mark, A.F., Christiani, G., Sigle, W., Habermeier, H.U., van Aken, P.A., et al.: Comparative study of $\mathrm{LaNiO}_{3} / \mathrm{LaAlO}_{3}$ heterostructures grown by pulsed laser deposition and oxide molecular beam epitaxy. Appl. Phys. Lett. 110, 041606 (2017). https://doi.org/10.1063/1.4975005

40. Lagally, M.G., Savage, D.E.: Quantitative electron diffraction from thin films. MRS Bull. 18, 24-31 (1993). https://doi.org/10. 1557/S0883769400043414

41. Bozovic, I., Eckstein, J.N.: Analysis of growing films of complex oxides by RHEED. MRS Bull. 20, 32-38 (1995). https://doi.org/ 10.1557/S0883769400044870

42. Herman, M.A., Sitter, H.: Molecular Beam Epitaxy: Fundamentals and Current Status. Springer-Verlag, Berlin Heidelberg (1989)

43. Kawamura, T., Maksym, P.A.: RHEED from stepped surfaces and its relation to RHEED intensity oscillations observed during MBE. Surf. Sci. 161, 12-24 (1985). https://doi.org/10.1016/ 0039-6028(85)90724-1

44. Grazing Incidence Configurations: Thin Film Analysis by X-Ray Scattering, pp. 143-182. John Wiley \& Sons, Ltd (2006). https:// doi.org/10.1002/3527607595.ch4

45. Bowen, D.K., Tanner, B.K., Tanner, B.K.: High Resolution X-Ray Diffractometry and Topography. CRC Press (1998). https://doi. org/10.1201/b12575.

46. Pickett, W.E.: Electronic structure of the high-temperature oxide superconductors. Rev. Mod. Phys. 61, 433-512 (1989). https:// doi.org/10.1103/RevModPhys.61.433

47. Smadici, S., Lee, J.C.T., Wang, S., Abbamonte, P., Logvenov, G., Gozar, A., et al.: Superconducting transition at $38 \mathrm{~K}$ in insulatingoverdoped $\mathrm{La}_{2} \mathrm{CuO}_{4}-\mathrm{La}_{1.64} \mathrm{Sr}_{0.36} \mathrm{CuO}_{4}$ superlattices: evidence for interface electronic redistribution from resonant soft X-ray scattering. Phys. Rev. Lett. 102, 107004 (2009). https://doi.org/10.1103/ PhysRevLett.102.107004

48. Benckiser, E., Haverkort, M.W., Brück, S., Goering, E., Macke, S., Frañó, A., et al.: Orbital reflectometry of oxide heterostructures. Nat. Mater. 10, 189-193 (2011). https://doi.org/10.1038/ nmat2958

49. Ament, L.J.P., van Veenendaal, M., Devereaux, T.P., Hill, J.P., van den Brink, J.: Resonant inelastic x-ray scattering studies of elementary excitations. Rev. Mod. Phys. 83, 705-767 (2011). https://doi.org/10.1103/RevModPhys.83.705

50. Hepting, M., Minola, M., Frano, A., Cristiani, G., Logvenov, G., Schierle, E., et al.: Tunable charge and spin order in $\mathrm{PrNiO}_{3}$ thin films and superlattices. Phys. Rev. Lett. 113, 227206 (2014). https://doi.org/10.1103/PhysRevLett.113.227206

51. Gázquez, J., Sánchez-Santolino, G., Biškup, N., Roldán, M.A., Cabero, M., Pennycook, S.J., et al.: Applications of STEMEELS to complex oxides. Mater. Sci. Semicond. Process. 65, 49-63 (2017). https://doi.org/10.1016/j.mssp.2016.06.005

52. Damascelli, A., Hussain, Z., Shen, Z.-X.: Angle-resolved photoemission studies of the cuprate superconductors. Rev. Mod. Phys. 75, 473-541 (2003). https://doi.org/10.1103/RevModPhys.75.473

53. Lanzara, A., Bogdanov, P.V., Zhou, X.J., Kellar, S.A., Feng, D.L., Lu, E.D., et al.: Evidence for ubiquitous strong electron-phonon coupling in high-temperature superconductors. Nature. 412, 510 (2001). https://doi.org/10.1038/35087518

54. Varela, M., Lupini, A.R., van, B.K., Borisevich, A.Y., Chisholm, M.F., Shibata, N., et al.: Materials characterization in the aberration-corrected scanning transmission electron microscope. Annu. Rev. Mater. Res. 35, 539-569 (2005). https://doi.org/10. 1146/annurev.matsci.35.102103.090513

55. MacLaren, I., Ramasse, Q.M.: Aberration-corrected scanning transmission electron microscopy for atomic-resolution studies of functional oxides. Int. Mater. Rev. 59, 115-131 (2014). https://doi.org/10.1179/1743280413Y.0000000026

56. Suyolcu, Y.E., Wang, Y., Baiutti, F., Sigle, W., Cristiani, G., Gregori, G., et al.: Aberration-corrected scanning transmission electron microscopy of $\mathrm{La}_{2} \mathrm{CuO}_{4}$-based superconducting interfaces at the Stuttgart Center for Electron Microscopy. JEOL News. 53, 8-17 (2018)

57. Schlom, D.G., Chen, L.-Q., Eom, C.-B., Rabe, K.M., Streiffer, S.K., Triscone, J.-M.: Strain tuning of ferroelectric thin films. Annu. Rev. Mater. Res. 37, 589-626 (2007). https://doi.org/10. 1146/annurev.matsci.37.061206.113016

58. Becher, C., Maurel, L., Aschauer, U., Lilienblum, M., Magén, C., Meier, D., et al.: Strain-induced coupling of electrical polarization and structural defects in $\mathrm{SrMnO}_{3}$ films. Nat. Nanotechnol. 10, 661-665 (2015). https://doi.org/10.1038/nnano.2015.108

59. Chandrasena, R.U., Yang, W., Lei, Q., Delgado-Jaime, M.U., Wijesekara, K.D., Golalikhani, M., et al.: Strain-engineered oxygen vacancies in $\mathrm{CaMnO}_{3}$ thin films. Nano Lett. 17, 794-799 (2017). https://doi.org/10.1021/acs.nanolett.6b03986

60. Agrawal, P., Guo, J., Yu, P., Hébert, C., Passerone, D., Erni, R., et al.: Strain-driven oxygen deficiency in multiferroic $\mathrm{SrMnO}_{3}$ thin films. Phys. Rev. B. 94, 104101 (2016). https://doi.org/10. 1103/PhysRevB.94.104101

61. Vailionis, A., Boschker, H., Siemons, W., Houwman, E.P., Blank, D.H.A., Rijnders, G., et al.: Misfit strain accommodation in epitaxial $\mathrm{ABO}_{3}$ perovskites: lattice rotations and lattice modulations. Phys. Rev. B. 83, 064101 (2011). https://doi.org/10.1103/ PhysRevB.83.064101

62. Bagués, N., Santiso, J., Esser, B.D., Williams, R.E.A., McComb, D.W., Konstantinovic, Z., et al.: The misfit dislocation core phase in complex oxide heteroepitaxy. Adv. Funct. Mater. (n.d.). https:// doi.org/10.1002/adfm.201704437

63. Naito, M., Sato, H.: Stoichiometry control of atomic beam fluxes by precipitated impurity phase detection in growth of (Pr, $\mathrm{Ce})_{2} \mathrm{CuO}_{4}$ and $\left(\mathrm{La}, \mathrm{Sr}_{2} \mathrm{CuO}_{4}\right.$ films. Appl. Phys. Lett. 67, 25572559 (1995). https://doi.org/10.1063/1.114431

64. Bozovic, I., Logvenov, G., Verhoeven, M.A.J., Caputo, P., Goldobin, E., Geballe, T.H.: No mixing of superconductivity and antiferromagnetism in a high-temperature superconductor. Nature. 422, 873-875 (2003). https://doi.org/10.1038/ nature 01544 . 
65. Keimer, B., Kivelson, S.A., Norman, M.R., Uchida, S., Zaanen, J.: From quantum matter to high-temperature superconductivity in copper oxides. Nature. 518, 179-186 (2015). https://doi.org/10. 1038/nature14165

66. Radaelli, P.G., Hinks, D.G., Mitchell, A.W., Hunter, B.A., Wagner, J.L., Dabrowski, B., et al.: Structural and superconducting properties of $\mathrm{La}_{2-\mathrm{x}} \mathrm{Sr}_{\mathrm{x}} \mathrm{CuO}_{4}$ as a function of $\mathrm{Sr}$ content. Phys. Rev. B. 49, 4163-4175 (1994). https://doi.org/10. 1103/PhysRevB.49.4163

67. Hashimoto, M., Vishik, I.M., He, R.-H., Devereaux, T.P., Shen, Z.-X.: Energy gaps in high-transition-temperature cuprate superconductors. Nat. Phys. 10, $483-495$ (2014). https://doi.org/10. 1038/nphys3009

68. Taillefer, L.: Scattering and pairing in cuprate superconductors. Ann. Rev. Condens. Matter Phys. 1, 51-70 (2010). https://doi. org/10.1146/annurev-conmatphys-070909-104117

69. Maier, J., Pfundtner, G.: Defect chemistry of the high-Tc superconductors. Adv. Mater. 3, 292-297 (1991). https://doi.org/10. 1002/adma.19910030605

70. Sato, H., Tsukada, A., Naito, M., Matsuda, A.: $\mathrm{La}_{2-\mathrm{x}} \mathrm{Sr}_{\mathrm{x}} \mathrm{CuO}$ y epitaxial thin films ( $\mathrm{x}=0$ to 2 ): structure, strain, and superconductivity. Phys. Rev. B. 61, 12447-12456 (2000). https://doi.org/10. 1103/PhysRevB.61.12447

71. Moodenbaugh, A.R., Sabatini, R.L., Xu, Y., Ochab, J., Huber, J.G.: Solubility of $\mathrm{Ca}$ in superconducting $\mathrm{La}_{2-x} \mathrm{Ca}_{\mathrm{x}} \mathrm{CuO}_{4}$. Physica C. 198, 103-108 (1992). https://doi.org/10.1016/09214534(92)90272-E

72. Shen, L., Salvador, P., Mason TO, Fueki, K.: High temperature electrical properties and defect chemistry of $\mathrm{La}_{2-\mathrm{x}} \mathrm{Ca}_{\mathrm{x}} \mathrm{CuO}_{4-\mathrm{y}}$ superconductors - II. Defect structure modeling. J. Phys. Chem. Solids. 57, 1977-1987 (1996). https://doi.org/10.1016/S00223697(95)00324-X

73. Naito, M., Tsukada, A., Greibe, T., Sato, H.: Phase control in La214 epitaxial thin films. Int. Soc. Opt. Photon. 4811, 140-155 (2002). https://doi.org/10.1117/12.455498

74. Sato, H., Tsukada, A., Naito, M., Matsuda, A.: Absence of 1/8 anomaly in strained thin films of $\mathrm{La}_{2-\mathrm{x}} \mathrm{Ba}_{\mathrm{x}} \mathrm{CuO}_{4}$. Phys. Rev. B. 62, R799-R802 (2000). https://doi.org/10.1103/PhysRevB.62.R799

75. Bozovic, I., Logvenov, G., Belca, I., Narimbetov, B., Sveklo, I.: Epitaxial strain and superconductivity in $\mathrm{La}_{2-\mathrm{x}} \mathrm{Sr}_{\mathrm{x}} \mathrm{CuO}_{4}$ thin films. Phys. Rev. Lett. 89, 107001 (2002). https://doi.org/10.1103/ PhysRevLett.89.107001

76. Suyolcu, Y.E., Wang, Y., Baiutti, F., Al-Temimy, A., Gregori, G., Cristiani, G., et al.: Dopant size effects on novel functionalities: high-temperature interfacial superconductivity. Sci. Rep. 7, 453 (2017). https://doi.org/10.1038/s41598-017-00539-4

77. Kao, H.L., Kwo, J., Fleming, R.M., Hong, M., Mannaerts, J.P.: In situ growth and properties of single-crystalline-like $\mathrm{La}_{2-\mathrm{x}} \mathrm{Sr}_{\mathrm{x}} \mathrm{CuO}_{4}$ epitaxial films by off-axis sputtering. Appl. Phys. Lett. 59, 2748 (1998). https://doi.org/10.1063/1.105876.

78. Wells, B.O., Birgeneau, R.J., Chou, F.C., Endoh, Y., Johnston, D.C., Kastner, M.A., et al.: Intercalation and staging behavior in super-oxygenated $\mathrm{La}_{2} \mathrm{CuO}_{4+\delta}$. Zeitschrift Für Physik B Condens. Matter. 100, 535-545 (1996). https://doi.org/10.1007/ s002570050158.

79. Shen, L., Salvador, P.A., Mason TO: Point defect modeling of $\mathrm{La}_{2} \mathrm{CuO}_{4}$-based superconductors. J. Am. Ceram. Soc. 77, 81-88 (1994). https://doi.org/10.1111/j.1151-2916.1994.tb06960.x

80. Torrance, J.B., Tokura, Y., Nazzal, A.I., Bezinge, A., Huang, T.C., Parkin, S.S.P.: Anomalous disappearance of high- $T_{c}$ superconductivity at high hole concentration in metallic $\mathrm{La} 2-\mathrm{xSxCuO} 4$. Phys. Rev. Lett. 61, 1127-1130 (1988). https://doi.org/10.1103/ PhysRevLett.61.1127

81. Opila, E.J., Tuller, H.L., Wuensch, B.J., Maier, J.: Oxygen tracer diffusion in $\mathrm{La}_{2-\mathrm{x}} \mathrm{Sr}_{\mathrm{x}} \mathrm{CuO}_{4-\mathrm{y}}$ single crystals. J. Am. Ceram. Soc.
76, 2363-2369 (1993). https://doi.org/10.1111/j.1151-2916.1993. tb07778.x

82. Cordero, F., Cantelli, R.: Interstitial $\mathrm{O}$ and $\mathrm{O}$ vacancies in $\mathrm{La}_{2} \mathrm{CuO}_{4+\delta}$ during high-temperature treatments. Physica C: Superconductivity. 312, 213-224 (1999). https://doi.org/10. 1016/S0921-4534(98)00712-6

83. Ray, P.J., Andersen, N.H., Jensen, T.B.S., Mohottala, H.E., Niedermayer, C., Lefmann, K., et al.: Staging superstructures in high-Tc Sr/O codoped $\mathrm{La}_{2-\mathrm{x}} \mathrm{Sr}_{\mathrm{x}} \mathrm{CuO}_{4+\mathrm{y}}$. Phys. Rev. B. 96, 174106 (2017). https://doi.org/10.1103/PhysRevB.96.174106

84. Kim, G., Christiani, G., Logvenov, G., Choi, S., Kim, H.-H., Minola, M., et al.: Selective formation of apical oxygen vacancies in $\mathrm{La}_{2-\mathrm{x}} \mathrm{Sr}_{\mathrm{x}} \mathrm{CuO}_{4}$. Phys Rev Materials. 1, 054801 (2017). https:// doi.org/10.1103/PhysRevMaterials.1.054801

85. Shannon, R.D.: Revised effective ionic radii and systematic studies of interatomic distances in halides and chalcogenides. Acta Cryst A. 32, 751-767 (1976). https://doi.org/10.1107/ S0567739476001551

86. Gozar, A., Bozovic, I.: High temperature interface superconductivity. Physica C: Superconductivity and Its Applications. 521522, 38-49 (2016). https://doi.org/10.1016/j.physc.2016.01.003

87. Božović, I., He, X., Wu, J., Bollinger, A.T.: Dependence of the critical temperature in overdoped copper oxides on superfluid density. Nature. 536, 309-311 (2016). https://doi.org/10.1038/ nature 19061

88. Leng, X., Božović, I.: Controlling superconductivity in $\mathrm{La}_{2-}$ ${ }_{\mathrm{x}} \mathrm{Sr}_{\mathrm{x}} \mathrm{CuO}_{4+\delta}$ by ozone and vacuum annealing. J. Supercond. Nov. Magn. 28, 71-74 (2015). https://doi.org/10.1007/s10948-0142888-2

89. Birgeneau, R.J., Christianson, R.J., Endoh, Y., Kastner, M.A., Lee, Y.S., Shirane, G., et al.: Structures and incommensurate spin excitations in excess oxygen-doped $\mathrm{La}_{2} \mathrm{CuO}_{4+\mathrm{y}}$. Phys. B Condens. Matter. 237-238, 84-90 (1997). https://doi.org/10.1016/S09214526(97)00058-6

90. Frano, A., Bluschke, M., Xu, Z., Frandsen, B., Lu, Y., Yi, M., et al.: Control of oxygen ordering in cuprate thin-film structures by electrochemistry. Phys. Rev. Mater. (n.d.)

91. Kaya, P., Gregori, G., Baiutti, F., Yordanov, P., Suyolcu, Y.E., Cristiani, G., et al.: High-temperature thermoelectricity in $\mathrm{LaNiO}_{3}-\mathrm{La}_{2} \mathrm{CuO}_{4}$ heterostructures. ACS Appl. Mater. Interfaces. 10, 22786 (2018). https://doi.org/10.1021/acsami. 8 b02153

92. Jia, C.L., Zeng, X.H., Xi, X.X., Urban, K.: Microstructure and residual strain in $\mathrm{La}_{2} \mathrm{CuO}_{4}$ thin films on $\mathrm{LaSrAlO}_{4}$-buffered $\mathrm{SrTiO}_{3}$ substrates. Phys. Rev. B. 64, 075416 (2001). https://doi. org/10.1103/PhysRevB.64.075416

93. Suyolcu, Y.E., Wang, Y., Sigle, W., Baiutti, F., Cristiani, G., Logvenov, G., et al.: Octahedral distortions at high-temperature superconducting $\mathrm{La}_{2} \mathrm{CuO}_{4}$ interfaces: visualizing Jahn-Teller effects. Adv. Mater. Interfaces. 4, 1700737 (2017). https://doi.org/ 10.1002/admi.201700737

94. Logvenov, G., Gozar, A., Bozovic, I.: High-temperature superconductivity in a single copper-oxygen plane. Science. 326, 699-702 (2009). https://doi.org/10.1126/science.1178863

95. Logvenov, G., Gozar, A., Butko, V.Y., Bollinger, A.T., Bozovic, N., Radovic, Z., et al.: Comprehensive study of high-Tc interface superconductivity. J. Phys. Chem. Solids. 71, 1098-1104 (2010). https://doi.org/10.1016/j.jpcs.2010.03.014

96. Pereiro, J., Bollinger, A.T., Logvenov, G., Gozar, A., Panagopoulos, C., Božović, I.: Insights from the study of hightemperature interface superconductivity. Philos. Trans. R. Soc. A Math. Phys. Eng. Sci. 370, 4890-4903 (2012). https://doi.org/10. 1098/rsta.2012.0219

97. Butko, V.Y., Logvenov, G., Božović, N., Radović, Z., Božović, I.: Madelung strain in cuprate superconductors - a route to 
enhancement of the critical temperature. Adv. Mater. 21, 3644 3648 (2009). https://doi.org/10.1002/adma.200803850

98. Sato, H., Naito, M.: Increase in the superconducting transition temperature by anisotropic strain effect in (001) $\mathrm{La}_{1.85} \mathrm{Sr}_{0.15} \mathrm{CuO}_{4}$ thin films on $\mathrm{LaSrAlO}_{4}$ substrates. Physica C: Superconductivity. 274, 221-226 (1997). https://doi.org/10.1016/ S0921-4534(96)00675-2

99. Zhou, H., Yacoby, Y., Butko, V.Y., Logvenov, G., Božović, I., Pindak, R.: Anomalous expansion of the copper-apical-oxygen distance in superconducting cuprate bilayers. PNAS. 107, 81038107 (2010). https://doi.org/10.1073/pnas.0914702107

100. Yacoby, Y., Zhou, H., Pindak, R., Božović, I.: Atomic-layer synthesis and imaging uncover broken inversion symmetry in $\mathrm{La}_{2}$ ${ }_{\mathrm{x}} \mathrm{Sr}_{\mathrm{x}} \mathrm{CuO}_{4}$ films. Phys. Rev. B. 87, 014108 (2013). https://doi. org/10.1103/PhysRevB.87.014108

101. Radović, Z., Božović, N., Božović, I.: Photoinduced expansion of cuprate superconductors: evidence of strong electron-lattice coupling. Phys. Rev. B. 77, 092508 (2008). https://doi.org/10.1103/ PhysRevB.77.092508

102. Ino, A., Kim, C., Mizokawa, T., Shen, Z.-X., Fujimori, A., Takaba, M., et al.: Fermi surface and band dispersion in $\mathrm{La} 2-\mathrm{xSrxCuO} 4$. J. Phys. Soc. Jpn. 68, 1496-1499 (1999). https://doi.org/10.1143/ JPSJ.68.1496.

103. Wu, J., Pelleg, O., Logvenov, G., Bollinger, A.T., Sun, Y.-J., Boebinger, G.S., et al.: Anomalous independence of interface superconductivity from carrier density. Nat. Mater. 12, 877-881 (2013). https://doi.org/10.1038/nmat3719

104. Radović, Z., Vanević, M., Wu, J., Bollinger, A.T., Božović, I.: Interface superconductivity in cuprates defies Fermi-liquid description. J. Supercond. Nov. Magn. 30, 1-5 (2016). https://doi. org/10.1007/s10948-016-3636-6

105. Baiutti, F., Logvenov, G., Gregori, G., Cristiani, G., Wang, Y., Sigle, W., et al.: High-temperature superconductivity in spacecharge regions of lanthanum cuprate induced by twodimensional doping. Nat. Commun. 6, 8586 (2015). https://doi. org/10.1038/ncomms 9586

106. Wang, Y., Baiutti, F., Gregori, G., Cristiani, G., Salzberger, U., Logvenov, G., et al.: Atomic-scale quantitative analysis of lattice distortions at interfaces of two-dimensionally $\mathrm{Sr}$-doped $\mathrm{La}_{2} \mathrm{CuO}_{4}$ superlattices. ACS Appl. Mater. Interfaces. 8, 6763-6769 (2016). https://doi.org/10.1021/acsami.5b12813

107. Baiutti, F., Gregori, G., Wang, Y., Suyolcu, Y.E., Cristiani, G., van Aken, P.A., et al.: Cationic redistribution at epitaxial interfaces in superconducting two-dimensionally doped lanthanum cuprate films. ACS Appl. Mater. Interfaces. 8, 27368-27375 (2016). https://doi.org/10.1021/acsami.6b09739.

108. Suyolcu, Y.E., Wang, Y., Sigle, W., Cristiani, G., Logvenov, G., van, A.P.A.: Linking dopant distribution and interatomic distortions at $\mathrm{La}_{1.6} \mathrm{M}_{0.4} \mathrm{CuO}_{4} / \mathrm{La}_{2} \mathrm{CuO}_{4}$ superconducting interfaces. Microsc. Microanal. 22, 308-309 (2016). https://doi.org/10. 1017/S1431927616002397

109. Anisimov, V.I., Korotin, M.A., Zaanen, J., Andersen, O.K.: Spin bags, polarons, and impurity potentials in $\mathrm{La}_{2-\mathrm{x}} \mathrm{Sr}_{\mathrm{x}} \mathrm{CuO}_{4}$ from first principles. Phys. Rev. Lett. 68, 345-348 (1992). https://doi.org/10. 1103/PhysRevLett.68.345

110. Lorenz, M., Rao, M.S.R., Venkatesan, T., Fortunato, E., Barquinha, P., Branquinho, R., et al.: The 2016 oxide electronic materials and oxide interfaces roadmap. J. Phys. D. Appl. Phys. 49, 433001 (2016). https://doi.org/10.1088/0022-3727/49/43/ 433001
111. Coll, M., Fontcuberta, J., Althammer, M., Bibes, M., Boschker, H., Calleja, A., et al.: Towards oxide electronics: a roadmap. Appl. Surf. Sci. 482, 1-93 (2019). https://doi.org/10.1016/j.apsusc.2019. 03.312

112. Baiutti, F., Gregori, G., Suyolcu, E.Y., Wang, Y., Cristiani, G., Sigle, W., et al.: High-temperature superconductivity at the lanthanum cuprate/lanthanum-strontium nickelate interface. Nanoscale. 10, 8712-8720 (2018). https://doi.org/10.1039/C8NR00885J

113. Kim, G., Khaydukov, Y., Bluschke, M., Suyolcu, Y.E., Cristiani, G., Weschke, E., et al.: Tunable perpendicular exchange bias in oxide heterostructures. Phys. Rev. Mater. 3, 084420 (2019). https://doi.org/10.1103/PhysRevMaterials.3.084420

114. De Luca, G.M., Ghiringhelli, G., Perroni, C.A., Cataudella, V., Chiarella, F., Cantoni, C., et al.: Ubiquitous long-range antiferromagnetic coupling across the interface between superconducting and ferromagnetic oxides. Nat. Commun. 5, 5626 (2014). https:// doi.org/10.1038/ncomms6626

115. Buzdin, A.I.: Proximity effects in superconductor-ferromagnet heterostructures. Rev. Mod. Phys. 77, 935-976 (2005). https:// doi.org/10.1103/RevModPhys.77.935

116. Efetov, K.B., Garifullin, I.A., Volkov, A.F., Westerholt, K.: Proximity effects in ferromagnet/superconductor heterostructures. In: Zabel, H., Bader, S.D. (eds.) Magnetic Heterostructures: Advances and Perspectives in Spinstructures and Spintransport, pp. 251-290. Springer Berlin Heidelberg, Berlin (2008). https:// doi.org/10.1007/978-3-540-73462-8 5.

117. Frano, A., Blanco-Canosa, S., Schierle, E., Lu, Y., Wu, M., Bluschke, M., et al.: Long-range charge-density-wave proximity effect at cuprate/manganate interfaces. Nat. Mater. 15, 831-834 (2016). https://doi.org/10.1038/nmat4682

118. May, S.J., Shah, A.B., te Velthuis, S.G.E., Fitzsimmons, M.R., Zuo, J.M., Zhai, X., et al.: Magnetically asymmetric interfaces in a $\mathrm{LaMnO}_{3} / \mathrm{SrMnO}_{3}$ superlattice due to structural asymmetries. Phys. Rev. B. 77, 174409 (2008). https://doi.org/10.1103/ PhysRevB.77.174409

119. Shah, A.B., Ramasse, Q.M., May, S.J., Kavich, J., Wen, J.G., Zhai, X., et al.: Presence and spatial distribution of interfacial electronic states in $\mathrm{LaMnO}_{3}-\mathrm{SrMnO}_{3}$ superlattices. Phys. Rev. B. 82, 115112 (2010). https://doi.org/10.1103/PhysRevB.82.115112

120. Bosman, M., Watanabe, M., Alexander, D.T.L., Keast, V.J.: Mapping chemical and bonding information using multivariate analysis of electron energy-loss spectrum images. Ultramicroscopy. 106, 1024-1032 (2006). https://doi.org/10.1016/j.ultramic. 2006.04.016

121. The use of MLLS fitting approach to resolve overlapping edges in the EELS spectrum at the atomic level | Gatan, Inc. n.d. http:// www.gatan.com/use-mlls-fitting-approach-resolve-overlappingedges-eels-spectrum-atomic-level. Accessed 11 Feb 2017

122. Wang, Y., Salzberger, U., Sigle, W., Eren Suyolcu, Y., van Aken, P.A.: Oxygen octahedra picker: a software tool to extract quantitative information from STEM images. Ultramicroscopy. 168, 4652 (2016). https://doi.org/10.1016/j.ultramic.2016.06.001

123. Wang, Y., Suyolcu, Y.E., Salzberger, U., Hahn, K., Srot, V., Sigle, W., et al.: Correcting the linear and nonlinear distortions for atomically resolved STEM spectrum and diffraction imaging. Microscopy (Oxf). 67, i114-i122 (2018). https://doi.org/10.1093/ jmicro/dfy002

Publisher's Note Springer Nature remains neutral with regard to jurisdictional claims in published maps and institutional affiliations. 